\title{
Interaction of Tearing Modes of Different Pitch in Cylindrical Geometry
}

\author{
H. Richard Hicks \\ Benjamin Carreras \\ Jeff A. Holmes \\ Bruce V. Waddell
}

OAK RIDGE NATIONAL LABORATORY OPERATED BY UNION CARBIDE CORPORATION - FOR THE DFPARTMENT OF ENERGY 


\section{DISCLAIMER}

This report was prepared as an account of work sponsored by an agency of the United States Government. Neither the United States Government nor any agency Thereof, nor any of their employees, makes any warranty, express or implied, or assumes any legal liability or responsibility for the accuracy, completeness, or usefulness of any information, apparatus, product, or process disclosed, or represents that its use would not infringe privately owned rights. Reference herein to any specific commercial product, process, or service by trade name, trademark, manufacturer, or otherwise does not necessarily constitute or imply its endorsement, recommendation, or favoring by the United States Government or any agency thereof. The views and opinions of authors expressed herein do not necessarily state or reflect those of the United States Government or any agency thereof. 


\section{DISCLAIMER}

Portions of this document may be illegible in electronic image products. Images are produced from the best available original document. 
Printed in the United States of America. Available from

National Technical Information Service

U.S. Department of Commerce

5285 Port Royal Road, Springfield, Virginia 22161

Price: Printed Copy $\$ 5.25$; Microfiche $\$ 3.00$

This report was prepared as an account of work sponsored by an agency of the United States Government. Neither the United States Government nor any agency thereof, nor any of their employees, contractors, subcontractors, or their employees, makes any warranty, express or implied, nor assumes any legal liability or responsibility for any third party's use or the results of such use of any information, apparatus, product or process disclosed in this report, nor represents that its use by such third party would not-infringe privately owned rights. 
Contract No. W-7405-eng-26

FUSION ENERGY DIVISION

\title{
INTERACTION OF TEARING MODES OF DIFFERENT PITCH
}

\section{IN CYLINDRICAL GEOMETRY}

\author{
H. Richard Hicks \\ Benjamin Carreras ${ }^{\star}$ \\ Jeff $A$. Holmes \\ Bruce V. Waddel 1
}

* On leave from Junta de Energia Nuclear, Madrid, Spain

Date Published - December 1977

NOTICE This document contains information of a preliminary nature.

It is subject to revision or çorrection and therefore does not represent a

final report.

\author{
Prepared by the \\ OAK RIDGE NATIONAL LABORATORY \\ Oak Ridge, Tennessee 37830 \\ operated by \\ UNION CARBIDE CORPORATION \\ for the \\ DEPARTMENT OF ENERGY
}


THIS PAGE

\section{WAS INTENTIONALLY LEFT BLANK}


CONTENTS

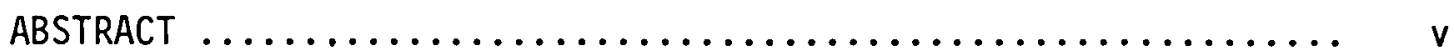

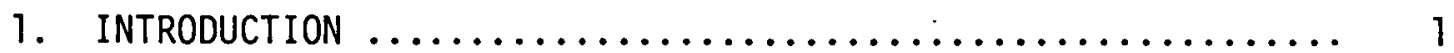

2. REDUCTION OF THE RESISTIVE MHD EQUATIONS $\ldots \ldots \ldots \ldots \ldots \ldots$.

3. NUMERICAL ALGORITHM FOR SOLVING THE REDUCED EQUATIONS ..... 16

4. NUMERICAL RESULTS FOR THE INTERACTION OF THE $m=2 / n=1$ AND $m=3 / n=2$ TEARING MODES $\ldots \ldots \ldots \ldots \ldots \ldots \ldots \ldots \ldots \ldots \ldots \ldots . .18$

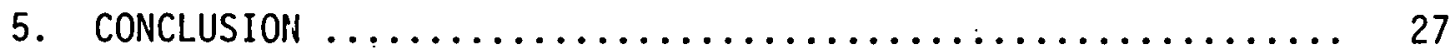

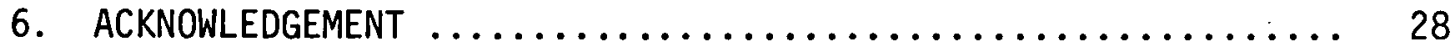

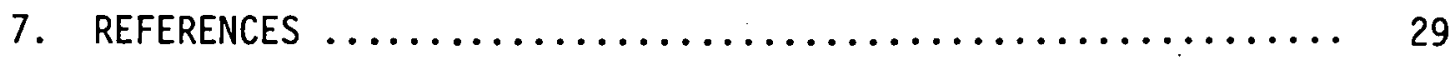




\section{THIS PAGE \\ WAS INTENTIONALLY \\ LEFT BLANK}




\section{ABSTRACT}

In cylindrical geometry, we analyze the hypothesis that the major disruption in tokamaks is due to the interaction of tearing modes of different pitch. For a flat safety factor profile, we find that the $m=3 / n=2$ tearing mode, which is linearly unstable, is strongly destabilized nonlinearly by the $m=2 / n=1$ mode. Other modes are nonlinearly destabilized, particularly the $m=1 / n=1$ and $m=5 / n=3$ modes. Due to the development of islands of many different pitches, the toroidal current density profile is severely deformed. The corresponding region of island activity can extend essentially from the plasma center to the limiter. Presumably, this deformation of the field lines can lead to rapid transport of heat and particles from the plasma center to the limiter. The destabilization of the $m=3 / n=2$ mode and other odd m modes is significant because it may correspond to the observed vertical asymmetry of some of the major disruptions in PLT. 


\section{INTRODUCTION}

The experimental limitation on plasma density and current in a tokamak is the major (or external) disruption.' In the most extreme cases, the major disruption is characterized by a radial expansion of the plasma column and a corresponding loss of confinement on a time scale of roughly $100 \mu \mathrm{sec}$. The most easily observed signature of a disruption is a negative voltage spike at the limiter. The precise circumstances preceding the disruption are quite varied, but one most of ten observes an increase in the $m=2 / n=1$ oscillations in the poloidal magnetic field at the limiter. Here, $m$ and $n$ denote the poloidal and toroidal mode numbers, respectively. Many times the $m=1$ and $m=2$ soft $x$-ray signals indicate that the radial expansion, i.e., the $m=0$ piece of the disruption, is centered at a surface inside the surface where the safety factor $q$ is 2 , perhaps the $q=3 / 2$ surface. $^{4}$ This last observation perhaps indicates the presence of an $m=3 / n=2$ instability because we expect that the most destructive instabilities have a pitch that matches the pitch of the field lines.

The consensus appears to be that the major disruption can be explained within the context of the MHD equations because the violence of the phenomenon probably indicates that some of the potential energy of the magnetic field is released; and thus these equations should reflect the basic physics. For the low mode number instabilities that are apparently involved, kinetic effects such as diamagnetic drifts, viscosity, and finite gyroradius corrections should merely modify the basic instability mechanism. 5,6 
Within the context of the MHD equations, it has often been suggested that tearing (or resistive kink) modes ${ }^{7,8}$ might be responsible for the major disruptions. From a theoretical viewpoint, low mode number tearing modes are the most likely candidates because ideal MHD surface kink modes are nonlinearly stabilized by shear ${ }^{9}$ and, except for $m=1$, the ideal internal kink modes are linearly stable in low $\beta$ plasmas. With regard to the $m=1$ instability, one observes that the linear growth rate of the ideal mode is typically less than that of the $m=1$ tearing mode. 5,10 Furthermore, the nonlinear treatment of Rosenbluth et al. Shows that the ideal $\mathrm{m}=1$ mode saturates at a relatively low amplitude. ${ }^{11}$

Another reason that tearing modes are serious candidates for the major disruption concerns the nonlinear growth of the $m=1$ tearing mode. In Refs. 12-15 the heuristic argument first presented by Kadomtsev ${ }^{16}$ was verified. In particular, it was shown that nonlinearly the $m=1$ tearing mode continues to exponentiate rapidly at essentially the linear growth rate until the safety factor, current density, and electron temperature profiles become flat inside the $q=1$ mode rational surface as magnetic reconnection occurs. ${ }^{14,17}$ Recent detailed comparisons of tearing mode theory with experimental data ${ }^{17}$ indicate that this process probably explains sawtooth oscillations (often referred to as internal or minor disruptions). These results stimulate one to consider mechanisms whereby tearing modes can cause magnetic reconnection to occur across the entire tokamak cross section instead of just the plasma core.

The difficulty with the argument that tearing modes are responsible for major disruptions is as follows. In Refs. 18-20 it was shown that for modes with $m>1$, once the width of the corresponding magnetic island 
exceeds the tearing layer width, the instability grows algebraically rather than exponentially on essentially the resistive time scale, which is on the order of milliseconds rather than microseconds. Furthermore, it has been shown that for many current profiles, the mode saturates at an amplitude corresponding to a magnetic island width of about $0.1 \mathrm{a}$, where $a$ is the minor radius. ${ }^{19,20}$ The recent results of White et al.; however, indicate that for safety factor profiles that are very flat in the plasma core, the $m=2$ island can continue to exponentiate rapidly to a width of approximately 0.7 a. ${ }^{21}$ Nevertheless, the results from our version of the MASS code, which will be presented in detail later in this report, show that the current density and the $m=2$ island quickly relax after the island reaches its maximum width; the final saturated width of the island is approximately $0.4 \mathrm{a}$. (It should be emphasized that this result was obtained without incorporating into the code the time evolution of the electron temperature; i.e., the resistivity was assumed to be independent of time.) Furthermore, the specific profile for which this result was obtained is unrealistic in that the safety factor at the plasma center $[q(0)]$ is 1.37 . One would expect that flat profiles are due to sawtooth activity which should maintain $q(0)$ at about unity. ${ }^{14}$ For such a flat profile, namely a profile with $q(0)=1.08$, there is no overshoot of the $m=2$ island and it saturates at about $0.4 \mathrm{a}$; this is probably insufficient to explain the major disruption, but it may correspond to the relatively large islands sometimes observed preceding a disruption. 22

In all of the analysis referred to in the preceding paragraph, however, the concept of helical symmetry ${ }^{9,16}$ was exploited and the 
resistive MHD equations were solved in cylindrical geometry. In view of the coupling between the $m=1$ and $m=2$ modes that is often observed, ${ }^{2,3,6}$ it is clearly desirable to study the resistive MHD in three dimensions and, in particular, to analyze the interaction of tearing modes of different pitch to determine if the interaction can cause rapid expansion of the plasma column. Furthermore, for the flat safety factor profiles of interest the linear growth rates of the $m=2 / n=1$ and $m=3 / n=2$ modes are comparable, but the two-dimensional (2-D) numerical code that was employed is obviously not capable of following the resulting strong coupling between modes of different pitch that one would expect to occur.

In this report we consider the nonlinear coupling of tearing modes of different pitch (as well as the same pitch) in cylindrical geometry. In order to study the problem numerically, we employ the standard tokamak ordering to derive a reduced set of resistive MHD equations valid for low $B$ (ratio of the plasma pressure to the magnetic pressure) that is subject to a reasonable restriction of the time step of the associated numerical scheme even for realistic values of the resistivity. This set of equations is a three-dimensional (3-D) version of the helically symmetric (twodimensional) set of equations derived in Ref. 9 and employed in Refs. 9, 12, 19, and 20. These three-dimensional equations have been employed by Strauss $^{23}$ with the resistive term omitted to study the nonlinear evolution of idea 7 MHD modes in rectangular geometry.

In this report, we present results from our three-dimensional code, referred to as RS3, for a flat safety factor profile with $q(0)=1.37$; here $q(0)$ denotes the safety factor at the plasma center. Results are presented for two values of $S$, the ratio of the skin time to the poloidal 
MHD time. In the nonlinear regime, we find that the $m=2 / n=1$ tearing mode can significantly destabilize the $m=3 / n=2$ mode. For $S=1.3 \times 10^{5}$ (defined at $r=0$ ), the instantaneous growth rate of the $m=3 / n=2$ flux perturbation increases by about a factor of four over the length of the run. Initially, the width of the magnetic island corresponding to the $m=3 / n=2$ perturbation is a factor of three smaller than the $m=2 / n=1$ island and the linear growth rate of the $m=3 / n=2$ is two-thirds that of the $m=2 / n=1$ mode. At the end of the run, however, the $m=3 / n=2$ island is as large as the $m=2 / n=1$ island (i.e., 0.35 a). In contrast, in a corresponding two-dimensional MASS code run, where the $m=2 / n=1$. mode is not present, the $m=3 / n=2$ island saturates at approximately. 0.1 a.

Furthermore, at the end of the run, the radial extent of the region encompassed by both the $m=2 / n=1$ and $m=3 / n=2$ islands is about 0.5 a; at the same time, the width of the $m=2$ magnetic island in the twodimensional run is about $0.35 \mathrm{a}$. In addition to the $m=3 / n=2$ mode, the $m=5 / n=3$ mode is also significantly destabilized, and at the end of the run the corresponding island width is approximately $0.15 \mathrm{a}$. The $\mathrm{m}=1 / \mathrm{n}=1$ mode is also destabilized.

In general, sharp deformations in the toroidal current density develop near the $x$-points (or saddle points) of magnetic islands. In the threedimensional code, several islands are present and the deformations in the current density tend to overlap and produce even sharper deformations. For large $S$, these sharper deformations limit the length of time the 3-D code can be run to times shorter than those of corresponding 2-D code runs. Consequently, we have also studied the same flat safety factor profile with $S=2 \times 10^{4}$ instead of $1.3 \times 10^{5}$. We find the same destabilization of the 
$m=3 / n=2$ by the $m=2 / n=1$. At the termination of the run, however, the deformation of the toroidal current density is more severe. Furthermore, many islands of substantial size develop with the region of island activity extending essentially from the plasma center to the limiter.

These results are theoretically and experimentally significant for several reasons. First, the importance of the $m=3 / n=2$ mode for flat profiles, as reflected in the linear growth rate, is clearly established nonlinearly. Second, the nonlinear acceleration of the growth of the $m=3 / n=2$ mode observed in the code may provide an explanation of the fact that the radial expansion of the plasma column in PLT is often observed to be centered about a surface that is inside the $q=2$ surface and perhaps coincides with the $q=3 / 2$ surface. ${ }^{4}$ Furthermore, the experimentally observed vertical asymmetry in the disruption ${ }^{4}$ could be explained by the presence of an $m=3 / n=2$ mode. There is evidence that the asymmetry is due to an $m=3 / n=2$ rather than to an $m=1 / n=1$ disturbance because the frequency is twice that of the $m=2 / n=1$ precursors. 4 The argument is as follows. If the mode is rotating in the toroidal direction, then the frequency is proportional to the toroidal mode number. On the other hand, if the mode is rotating in the poloidal direction, the frequency should be proportional to the poloidal mode number. The frequency can be explained by an $m=3 / n=2$ mode rotating toroidally; however, it cannot be explained by an $m=1 / n=1$ mode rotating in either direction.

The body of this report is organized as follows: in Sect. 2, the three-dimensional set of reduced MHD equations is derived; in Sect. 3 , the numerical scheme employed to advance the equations is described; in Sect. 4, 
numerical results from RS3 are presented for flat q.profile with $q(0)=$ 1.37; and in Sect. 5 some concluding remarks are made. 


\section{REDUCTION OF THE RESISTIVE MHD EQUATIONS}

In MKS units, the usual resistive fluid equations are

$$
\begin{aligned}
& \rho \frac{d \underline{V}}{d t}=-\underline{\nabla P}+\underline{J} \times \underline{B}, \\
& \underline{J}=\underline{\nabla} \times B / \mu_{0}, \\
& \frac{\partial B}{\partial t}=-\underline{\nabla} \times \underline{E},
\end{aligned}
$$

and

$$
\underline{[}+\underline{V} \times \underline{B}=n \underline{J} .
$$

Here $\rho$ is the mass density, $d / d t$ is the total or convective time derivative, $\underline{V}$ is the fluid velocity, $P$ is the pressure, $\underline{J}$ is the current density, $\mu_{0}$ is the vacuum magnetic permeability, $\underline{B}$ is the magnetic intensity, $\underline{E}$ is the electric intensity, and $n$ is the resistivity. In order to close this set of equations, an equation of state, an equation for the mass density, and an equation for the resistivity must be specified. For the purpose of the following derivation, we may assume that the resistivity is a specified function of space and time. The equation of state will be discussed later in this section.

In order to reduce these equations to a readily tractable threedimensional set, we exploit the standard tokamak inverse aspect ratio ordering; i.e., we assume that $\varepsilon \equiv a / R_{0} \ll 1$, where $a$ and $R_{0}$ are respectively the minor and major radii of the torus, and thereby eliminate the fast MHD time scale associated with the Alfven waves propagating in the poloidal direction. The technique employed is similar to that used in Ref. 9 to derive the helically symmetric (two-dimensional) equations. 
Although we shall eventually ignore all toroidal effects in this report, we employ the usual toroidal coordinate system, denoting the radial, poloidal, and toroidal coordinate by $r, \theta$, and $\zeta$, respectively. First, we observe that the perturbation in the magnetic field should be small for the problems of interest. Specifically,

$$
\tilde{\mathrm{B}}_{\zeta} \leqslant\left|\underline{\tilde{B}}_{\perp}\right| \leqslant \varepsilon\left|\underline{\mathrm{B}}_{10}\right| \sim \varepsilon^{2} \mathrm{~B}_{\zeta 0},
$$

where the " $\sim$ " and " 0 " subscript denote perturbed and unperturbed quantities, respectively and the " $\perp$ " subscript denotes vectors perpendicular to $\zeta$. Consequently, to first order in $\varepsilon$, the perturbation in the toroidal magnetic field can be neglected. This means that we automatically eliminate the "fast" MHD time scale, i.e., the time scale for the propagation of the Alfvén waves across the magnetic field, namely $\tau_{H} \equiv a / V_{A}$, where $V_{A}$ is Alfvén speed. The remaining MHD time scale is the time for Alfuen waves to propagate along the magnetic field, given by $\tau_{H p}=R / V_{A}$, the so-called "slow" MHD time. Consequently, the time step restriction for numerical stability of any numerical scheme based on these equations will automatically be less severe than the time step restriction of a scheme which includes the time variation of $B_{\zeta}$.

Neglecting the time variation of $\mathrm{B}_{\zeta}$ also allows us to write the magnetic field in terms of the gradient of a scalar variable. The toroidal component of the equation $\underline{B}=\underline{\nabla} \times \underline{A}$ yields

$$
B_{\zeta}=B_{\zeta 0}=\frac{1}{r} \frac{\partial}{\partial r}\left(r A_{\theta}\right)-\frac{\partial}{\partial \theta} A_{r} ;
$$

here $\underline{A}$ is the usual vector potential. Since we are free to choose the gauge such that $A_{r}=0$, Eq. (5) implies that 


$$
A_{\theta}=\frac{1}{r} \int_{0}^{r} d r^{\prime} r^{-} B_{50}(r, \theta)
$$

Notice that since $B_{\zeta 0}$ is independent of $\zeta$ in an axisymmetric tokamak, $A_{\theta}$ is also independent of $\zeta$. Then, the radial and poloidal components of $\underline{B}=\underline{\nabla} \times \underline{A}$ imply that

$$
\underline{B}=-\frac{1}{R} \underline{\nabla} \times \hat{\zeta}+B_{\zeta 0} \hat{\zeta},
$$

where $\Psi \equiv-R A_{\zeta}$ is the standard poloidal flux function and $R=R_{0}+$ $r \cos \theta$. Notice that the magnetic field is described in terms of a scalar variable such that the requirement that $\underline{\nabla} \cdot \underline{B}=0$ is automatically satisfied, which means that any numerical scheme based on these equations will produce a magnetic field which also automatically satisfies this requirement.

An equation for the time evolution of the poloidal flux can be obtained by observing first that

$$
\frac{\partial A}{\partial t}=-\underline{E}+\underline{\nabla} \Phi=\underline{V} \times \underline{B}-\eta \underline{\underline{J}}+\underline{\nabla} \Phi,
$$

where $\Phi$ is a scalar function, sometimes referred to as the "electrostatic potential." Then, since $\hat{\zeta} \cdot(\underline{V} \times \underline{B})=\left(V_{\perp} \cdot \underline{\nabla} \Psi\right) / R$, the toroidal component of Eq. (8) yields

$$
\frac{D \Psi}{D t} \equiv \frac{\partial \Psi}{\partial t}+\underline{V}_{1} \cdot \underline{\nabla \Psi}=n R J_{\zeta}-\frac{\partial \Phi}{\partial \zeta}
$$

Notice that it is rigorously valid that the total or convective derivative appearing in the preceding equation does not involve the toroidal velocity. From Eq. (2), the toroidal current density appearing in Eq. (9) is given by 


$$
\mu_{0} J_{\zeta}=\frac{1}{R} \Delta^{*} \Psi \equiv \nabla_{\perp}^{2}(\Psi / R)+\Psi / R^{3},
$$

where $\zeta$ derivatives do not occur in $\Delta^{*}$, the so-called Laplacian operator in toroidal geometry.

The momentum balance equation can be used to show that the toroidal component of the fluid velocity can be neglected. We observe that because the safety factor $q$ is of order one in a tokamak, $J_{\zeta} \sim\left|\underline{B}_{1}\right| /\left(\mu_{0} a\right)$ and $\left|\underline{J}_{1}\right| \sim\left|\underline{B}_{1}\right| /\left(\mu_{0} R_{0}\right) \sim \varepsilon J_{\zeta}$. If. $\beta \sim \varepsilon^{2}$ and if the maximum perturbation in the plasma pressure does not exceed the unperturbed value, the toroidal and perpendicular components of Eq. (1) imply that

$$
\gamma \rho \tilde{V}_{\zeta} \sim \varepsilon^{3} B_{\zeta}^{2} / a
$$

and

$$
\gamma \rho\left|\tilde{V}_{1}\right| \sim \varepsilon^{2} B_{\zeta} / a
$$

where $\gamma$ is the instantaneous growth rate of the instability of interest. Equations (11) and (12) imply that the toroidal velocity can he neglected because

$$
\tilde{v}_{\zeta} \sim \varepsilon\left|\tilde{V}_{1}\right|
$$

Now, Eqs. (13) and (8) and the time independence of $B_{\zeta}$ can be used to. show that $\underline{V}_{-}$can be written in terms of a stream function, which turns out to be the "electrostatic potential" $\Phi_{\text {. }}$. Because $B_{\zeta}$ is constant in time, the poloidal components of A are also constant in time. Thus, the cross product of $\dot{\zeta}$ and Eq. (8) gives 


$$
\underline{v}_{-}=\frac{?}{B_{\zeta 0}} \nabla \Phi \times \hat{\zeta}+0\left(\varepsilon^{2}\right)
$$

Equation (14) implies that $\nabla \cdot\left(B_{50} V / R\right)=0$, which is the equation of state that is compatible with the low $\beta$ tokamak ordering.

Because the fluid velocity can be written in terms of a stream function, the momentum balance equation can be reduced to an equation for the vorticity. Ignoring spatial derivatives of $\rho$ and taking the toroidal component of the curl of Eq. (1), we obtain

$$
\rho \frac{D U}{D t}=-\frac{1}{R_{0}{ }^{2}} \hat{\zeta} \cdots\left(\nabla \Psi \times \underline{\nabla} J_{\zeta}\right)-\frac{B_{\zeta 0}}{R_{0}{ }^{2}} \frac{\partial J_{\zeta}}{\partial \zeta}+0(\varepsilon),
$$

where the vorticity $U$ is given by

$$
U=\nabla_{-1} \cdot\left(\frac{1}{R^{2}} \nabla_{-1} \Phi\right)
$$

We are also presently investigating the fully toroidal equations where the order $\varepsilon$ terms in Eq. (15) are retained. In deriving Eqs. (7), (9), (10), and (14), we kept the $r \cos \theta$ in $R$, but now in order to be consistent with the omission of the order $\varepsilon$ terms in Eq. (15), we must replace $R$ by $R_{0}$, take $B_{\zeta 0}$ to be constant in space, and neglect the $\Psi / R^{3}$ term in $J_{\zeta}$. Because we assume that the plasma fills the entire volume of the container, an equation for the magnetic field in the vacuum is unnecessary. The resistivity profile can be adjusted so that the resistivity is very large near the wall; for tokamak conditions, this approximation is probably better than the vacuum region approximation employed in ideal MHD theory. 
This reduced set of three-dimensional equations was previously derived for rectangular geometry with the resistivity term omitted and used to study the nonlinear evolution of the ideal MHD modes. ${ }^{23}$ If we were to assume that all quantitiés were functions only of $r$ and $\tau \equiv m \theta+n \zeta$ and if we used the helical flux function $\psi$ instead of the poloidal flux function $\psi$, where the two functions are related by $\psi=-\Psi / R_{0}-n r^{2} B_{\zeta} /\left(2 m R_{0}\right)$, we would obtain the helically symmetric equations derived in Ref. 9 and employed in Refs. 9, 12, and 14-21.

It is convenient to rewrite the reduced equations in dimensionless form. We normalize all the lengths to the minor radius $a$, the resistivity to some typical value $\bar{n}$, the time to the skin or resistive diffusion time: $\tau_{R}\left[\equiv a^{2} /\left(\mu_{0} \bar{n}\right)\right]$, the velocity to $a / \tau_{R}$, the flux to $a^{2} B_{\zeta 0}$, the magnetic field to $B_{0}$, and the current density to $B_{\zeta 0} /\left(\mu_{0} R_{0}\right)$. It is also convenient to replace $\psi$ by $\psi-E_{\zeta}^{W}$, where $E_{\zeta}^{W}$ is the constant electric field at the wall, so that the boundary condition on $\Psi$ becomes $\Psi(a, \theta, \zeta, t)=0$. Then the relevant equations become

$$
\begin{aligned}
& \underline{B}=-\varepsilon \nabla_{1} \Psi \times \hat{\zeta}+\hat{\zeta}, \\
& \frac{D \Psi}{D t}=\eta J_{\zeta}-E_{\zeta}^{W}-\frac{\partial \Phi}{\partial \zeta}, \\
& J_{\zeta}=\nabla_{\perp}^{2} \Psi, \\
& \underline{v}_{\perp}=\underline{\nabla}^{2} \times \hat{\zeta},
\end{aligned}
$$


and

$$
\frac{D U}{D t}=-S^{2}\left[\hat{\zeta} \cdot\left(\underline{\nabla} \times \underline{\nabla J}_{\zeta}\right)+\frac{\partial J_{\zeta}}{\partial \zeta}\right] .
$$

where $U=\nabla_{1}^{2} \Phi$ and $S \equiv \tau_{R} / \tau_{H p}$ with $\tau_{H p}=R_{0}\left(\mu_{0} \rho\right)^{1 / 2} / B_{\zeta 0}$ being the "slow" Alfvén time, i.e., the time for Alfvén waves to propagate around the machine in the toroidal direction. Notice that if we choose $E_{\zeta}^{W}$ and $n$ such that initially $E_{\zeta}^{W}=n_{\zeta}$, then $\Psi$ will not change if there is no tearing mode activity. Because the radial velocity must vanish at the wall, the appropriate boundary condition on $\Phi$ is $\Phi(a, \theta, \zeta)=0$.

It is also usefur to consider the energy integral of Eqs. (17)-(21). In terms of $\Phi$ and $\Psi$, the usual energy balance equation in dimensionless form becomes

$$
s^{-2} \frac{d E_{K}}{d t}+\frac{d E_{M}}{d t}=-Q_{J}+Q_{p},
$$

where

$$
\begin{aligned}
& E_{K} \equiv \frac{1}{2} \int_{V_{0}} d^{3} \underline{r}|\underline{\nabla} \Phi|^{2}, \\
& E_{M} \equiv \frac{1}{2} \int_{V_{0}} d^{3} \underline{r}|\underline{\nabla} \Psi|^{2}, \\
& Q_{J} \equiv \int_{V_{0}} d^{3} \underline{r} n J_{\zeta}{ }^{2},
\end{aligned}
$$

and

$$
Q_{p} \equiv \int_{V_{0}} d^{3} \underline{r} E_{\zeta}^{W} J_{\zeta}
$$


Here, $E_{K}$ is the kinetic energy, $E_{M}$ is the magnetic energy, $Q_{J}$ is the rate of energy dissipation due to Joule heating, and $Q_{p}$ is the Poynting flux through the wall written in terms of a volume integral. Notice that if we choose $n J_{\zeta}=E_{\zeta}^{W}$ initially, then the total energy of the system will not change if there is no tearing mode activity because the dissipation is balanced by the Poynting flux through the wall. 


\section{NUMERICAL ALGORITHM FOR SOLVING THE REDUCED EQUATIONS}

We observe that in the reduced equations the Laplacian operator that occurs has derivatives only in the poloidal plane so that the inversion of Poisson's equation to find $\Phi$ from $U$ is relatively simple: In addition, only first order $\zeta$ derivatives occur. These two facts greatly simplify the numerics and permit the construction of an algorithm that is similar to the one employed in the MASS code in Refs. 14, 19, and 21 and described in Ref. 24.

In order to simplify the notation, we define $S$ to be the right side of Eq. (21); $\delta / \delta r, \delta / \delta \theta$, and $\delta / \delta \zeta$ to be the finite difference forms of $\partial / \partial r,(1 / r)(\partial / \partial \theta)$, and $\partial / \partial \zeta$, respectively; and $\delta^{2} / \delta r^{2}$ and $\delta^{2} / \delta \theta$ to be the finite difference forms of the pieces of $\nabla_{1}{ }^{2}$ with radial and poloidal derivatives, respectively. We employ a two-step time advancement method whereby the vorticity is stepped explicitly and the flux equation is advanced implicitly using the alternating-direction method.

The first half of the time step is given by

$$
\begin{aligned}
& u^{t+\frac{1}{2}}=u^{t}+\frac{\Delta t}{2}\left(s^{t}-\underline{v}_{1}^{t} \cdot \underline{\nabla}_{1} u^{t}\right) \\
& \Psi^{t+\frac{1}{2}}=\Psi^{t}+\frac{\Delta t}{2}\left[-v_{r} \frac{\delta \Psi}{\partial r}+n \frac{\delta^{2} \Psi}{\delta r^{2}}-\frac{\delta \Phi}{\delta \zeta}\right]^{t+\frac{1}{2}}+\frac{\Delta t}{2}\left[-v_{\theta} \frac{\delta \Psi}{\delta \theta}+n \frac{\delta^{2} \psi}{\delta \theta^{2}}\right]^{t} .
\end{aligned}
$$

Once $U^{t+\frac{1}{2}}$ is determined from Eq. (22), $\Phi^{t+\frac{1}{2}}$ is determined by inverting Eq. (16); the Fourier transform technique employed in Refs. 9, 14, and 19 is used. Of course, once $\Phi$ is known, $\underline{v}_{1}^{t+\frac{1}{2}}$ can be obtained from Eq. (20). Then, $\psi^{t+\frac{1}{2}}$ can be found from Eq. (23), where the radial derivatives are implicit, by inverting a tridiagonal matrix. In the second half of 
the time step, which centers the time advancement, the poloidal derivatives are treated implicitly. Specifically, we have

$$
u^{t+l}=u^{t}+\Delta t\left(S^{t+\frac{1}{2}}-\underline{v}^{t+\frac{1}{2}} \cdot \nabla_{1} u^{t+\frac{1}{2}}\right)
$$

and

$$
\psi^{t+1}=\psi^{t+\frac{1}{2}}+\frac{\Delta t}{2}\left[-V_{r} \frac{\Delta \Psi}{\Delta r}+\eta \frac{\delta^{2} \psi}{\delta r^{2}}-\frac{\delta \Phi}{\delta \zeta}\right]^{t+\frac{1}{2}}+\frac{\Delta t}{2}\left[-V_{\theta} \frac{\delta \Psi}{\delta \theta}+\eta \frac{\delta^{2} \psi}{\delta \theta^{2}}\right]^{t+1} .
$$

As shown in Ref. 24, the time step limitation for this algorithm is essentially $\Delta t \leq(2 r \Delta \theta) /(|\underline{\nabla} \Psi|+r \Delta \theta / \Delta \zeta)$, where $\Delta \theta$ and $\Delta \zeta$ are the poloidal and toroidal grid spacings, respectively.

The computer code that advances the equations according to the preceding scheme is called RS3. The grid employed can be localized in the radial direction in order to support small scale radial variations, aithough for the runs presented in this report the grid was essentially uniform. Typically, the number of radial, poloidal, and toroidal grid points is 60,30 , and 15 , respectively. 
4. NUMERICAL RESULTS FOR THE INTERACTION OF THE $m=2 / n=1$ AND $m=3 / n=2$ TEARING MODES

The specific safety factor profile studied is the flat model introduced in Ref. 8 and given by

$$
q(r)=2\left[\frac{1+\left(r / r_{0}\right)^{8}}{1+\left(r_{s 2} / r_{0}\right)^{8}}\right]^{1 / 4} .
$$

We choose $r_{s 2}$, the position of the $q=2$ singular surface, to be $0.7 \mathrm{a}$ and $r_{0}$, the "width" of the current channel, to be $0.6 \mathrm{a}$; a plot of $q$ for these parameters is shown in Fig. 1. This profile is nearly flat for $r \leqslant 0.4$ a. The $q=3 / 2$ and $q=5 / 3$ surfaces are respectively located at $0.54 \mathrm{a}$ and $0.62 \mathrm{a}$. The resistivity profile is taken to be independent of time but with a spatial dependence given by $n(r)=J_{\zeta}\left(r_{s 2}\right) / J_{\zeta}(r)$. This functional form for the resistivity profile ensures that the system does not evolve in time (in particular, that the current channel does not decay) if there is no tearing mode activity.

The preceding safety factor profile is one of the profiles studied by White et al. ${ }^{21}$ with the two-dimensional MASS code. A summary of the two-dimensional results, which we have obtained with our version of the MASS code, is presented here.

The essential two-dimensional result is that for $S=1 \times 10^{4}[n(r)$ is such that the effective value of $S$ at $r=0$ is $6.4 \times 10^{4}$ ], the $m=2$ magnetic island, rather than slowing down in time and saturating at a relatively small size $(\approx 0.1$ a) as is the case for the peaked current profile, exponentiates rapidly until it reaches a maximum width of approximately $0.7 \dot{a}$ and then abruptly contracts and saturates at a 
width of 0.4 a. In Fig. 2, the magnetic island width $W$, normalized to the minor radius, is plotted as a function of time $t$, normalized to $\tau_{R}$, the resistive diffusion time. Notice that for this $S$-value, the growth rate of the magnetic island does not slow down and enter an algebraic growth regime where $d W / d t=n \Delta^{-}$; here $\Delta^{-}$is the quantity computed in the linear theory that is a measure of the discontinuity in the radial derivative of the eigenfunction across the singular layer and is proportional to the $\delta W$ of ideal MHD theory. ${ }^{25}$ Instead of entering the algebraic regime when $W$ exceeds the tearing layer width $\delta\left(\approx 2 \times 10^{-2}\right.$ a for $S=1 \times 10^{4}$ ), the mode continues to exponentiate until it reaches a maximum at $W \approx 0.7 \mathrm{a}$. The significance of the rapid growth is that rather than the process occuring on the millisecond time scale, it can occur in roughly $100 \mu \mathrm{sec}$. Whether or not the growth actually does slow down as $\delta$ decreases, i.e., $S$ increases, has not been established. After reaching a maximum width, the island abruptly contracts and saturates at $0.4 \mathrm{a}$.

In Fig. 3, where the helical flux contours are plotted at the times indicated by the arrows in Fig. 2, the dramatic expansion and contraction of the $m=2$ island can be observed.

In Fig. 4, the current density as a function of $r$ is plotted at the times indicated by the arrows in Fig. 2 (notice the change in the vertical scale with time). As indicated in Fig. 4, to the right of $r=0$, the current density is plotted along the ray that passes through the $x$-point of the island $(\theta=0)$; to the left of $r=0$, the current density is plotted along the ray that passes through the 0-point of the island $(\theta=\pi / 2)$. The deformation in the toroidal current density is particularly sharp or steep near the saddle point or $x$-point. The rcmarkable relaxation 
of the toroidal current density after the island reaches its maximum width can also be observed in Fig. 4. It should be emphasized that the contraction of the island and the relaxation of the current density may result from the procedure of choosing the resistivity profile to be independent of time. If the resistivity were advanced self-consistently through the electron heat transport equation, this effect might disappear.

A linear instability analysis of the profile in Fig. I, however, shows that the $m=3 / n=2$ and the $m=5 / n=3$ modes as well as the $m=2 /$ $n=1$ mode are unstable. Specifically, in resistive units, $\gamma^{21}=340$ and $\gamma^{32}=230$ for $S=2 \times 10^{4}$; here $\gamma$ is the growth rate $w i$ th the first and second superscripts respectively denoting the poloidal and toroidal mode numbers. Since the $m=5 / n=3$ is only slightly unstable, its growth cannot be accurately ascertained. The harmonics of the $m=2 / n=1$ and the $m=3 / n=2$ modes, particularly the $m=4 / n=2$ and $m=6 / n=2$ modes, are stable but near marginal stability. Because several modes of different helicity are clearly involved, the safety factor profile in Fig. 1 can only be studied with a fully three-dimensional code, such as RS3.

Before presenting the results from RS3, we present the results of our analysis of the $m=3 / n=2$ helicity using the two-dimensional MASS code. Rather than continuing to exponentiate rapidly as the $m=2 / n=1$ island, the growth of the $m=3 / n=2$ island slows down as it enters the nonlinear regime and saturates. In other words, the $m=3 / n=2$ mode behaves in the same way as the $m=2 / n=1$ for the peaked current profiles studied in Refs. 19 and 20 . We obtain the same result with RS3 when running the $m=3 / n=2$ helicity alone. 
In contrast to the two-dimensional run, we find that in the threedimensional RS3 run, the $m=3 / n=2$ mode is strongly destabilized by the presence of the $m=2 / n=1$. Furthermore, the $m=5 / n=3$ and $m=1 /$ $\mathrm{n}=1$ modes are generated and also strongly destabilized.

At the beginning of the run, we perturb only the $m=2 / n=1$ and the $m=3 / n=2$ modes. These two modes quickly become eigenmodes with the correct linear growth rates. For our choice of initial perturbations, the amplitude of the $m=2 / n=1$ is a factor of ten larger than the amplitude of the $m=3 / n=2$ mode. The $m=5 / n=3$ and other modes are generated nonlinearly. The value of $S$ is $2 \times 10^{4}$.

In Fig. 5, the maximum amplitude of the flux perturbations of various modes as obtained from RS3 are plotted as a function of time. First, we observe that the nonlinear growth rate of the $m=2 / n=1$ mode is slightly decreased from that in uncoupled runs. Second, rather than slowing down and saturating as in the uncoupled runs, the $m=3 / n=2$ mode continues to grow with an accelerating growth rate until at the end of the run its amplitude is only a factor of two smaller than the $m=2 / n=1$ mode. Third, the growth rate of the $m=5 / n=3$ mode also accelerates substantially. The increases in the growth rates of these modes are shown in Fig. 6 , where the instantaneous growth rate $\gamma$, defined as $[d(\max \tilde{\Psi}) / d t] /(\max \tilde{\psi})$, is plotted as a function of time. Notice that the growth rates peak near the end of the run at $t \approx 2.2 \times 10^{-2} \tau_{R}$. Although not shown in Fig. 6 , the growth rate of the $m=1 / n=1$ also accelerates and is essentially the same as that of the $m=5 / n=3$.

The instantaneous growth rate of the fluid kinetic energy of the conupled system also accelerates with time. We define the growth rate by 
$\left.\gamma \equiv\left(d E_{K} / d t\right) / 2 E_{K}\right)$, where $E_{K}$ is the total fluid kinetic energy; the factor of two is inserted so that when there is a single eigenmode, this growth rate will be the same as the growth rate of the flux eigenfunction in the linear regime. In Fig. 7, the kinetic energy growth rate for various runs is plotted as a function of time. The solid curve is the growth rate from RS3 when several different helicities are present. The dashed curve is the growth rate from the MASS code for the $m=2 / n=1$ helicity only. The dashed-dotted curve is the growth rate from the MASS code for the $m=3 / n=2$ helicity only. The dotted curve is also the growth rate for the $m=3 / n=2$ helicity only, but it was obtained from RS3 and thus serves to "benchmark" RS3 with the MASS code. The run corresponding to the dotted curve also verifies the fact that if only one helicity is excited initially then the system retains that helical symmetry throughout the length of the run. We observe that for $t \leqslant 1.5 \times 10^{-2} \tau_{R}$, the growth rate for the coupled or multihelicity system decreases in the same way that the growth rate in the single-helicity $m=2 / n=1$ run. At $t=1.5 \times 10^{-2} \tau_{R}$, however, the growth rate in the coupled system increases rapidly until it exceeds the growth rate near the beginning of the run. The growth rate in the single-helicity $m=2 / n=1$ run also increases but at a later time.

Figure 8 shows that the interaction of the modes of different helicity increases the total amount of kinetic energy associated with the fluid motion. The magnitude of the fluid kinetic energy for various runs is plotted as a function of time. The total kinetic energy of the coupled system exceeds by a factor of ten the sum of the total kinetic energy of the single-helicity $m=2 / n=1$ system and the total kinetic energy 
of the single-helicity $m=3 / n=2$ system. These results imply that the interaction of modes of different helicity increases the amount of energy released by the magnetic field.

When more than one helicity is involved, the description of the magnetic field lines is difficult because in general a flux function cannot be defined. In order to describe the interaction of magnetic islands, we employ the following technique. We expand the poloidal flux function in a Fourier series in $\theta$ and $\zeta$ and group the terms according to their helicity or pitch;

$$
\Psi=\psi_{00}+\sum_{p} \sum_{m / n=p} \tilde{\Psi}_{m n} \cos (m \theta+n \zeta),
$$

where the pitch $p$ is not necessarily an integer. Then, if a given helicity plus the $m=0 / n=0$ piece of $\psi$ is isolated, the helical flux function $\psi_{p}$ for that helicity can be defined by

$$
\psi_{p} \equiv-p\left[\Psi_{00}+\sum_{m / n=p} \tilde{\Psi}_{m n} \cos (m \theta+n \zeta)\right]-\frac{1}{2} r^{2} .
$$

In Figs. $9-11$, the contours of $\psi_{2 / 1}, \psi_{3 / 2}$, and $\psi_{5 / 3}$ in the $\zeta=0$ plane are plotted, at the times indicated in Figs. 5, 7, and 8 . In order to illustrate the overlap of the $m=2 / n=1$ and $m=3 / n=2$ magnetic islands, contours of $\psi_{2 / 1}$ are plotted in the upper half plane in Fig. 12 and $\psi_{3 / 2}$ in the lower half plane. The width of a magnetic island is defined as the maximum width along a ray of the separatrix contour. In Fig. 13, the widths of the $m=2 / n=1, m=3 / n=2$, and $m=5 / n=3$ is lands. are plotted as functions of time; the arrows indicate the times at which the flux contours in Figs. 9-12 are plotted. 
Several observations can be made concerning Figs. 9-13. First, the growth rate of the $m=2 / n=1$ island in the three-dimensional run decreases somewhat so that at the end of the run, the $m=2 / n=1$ island is about $25 \%$ smaller in the corresponding two-dimensional MASS code run. Second, the growth of the $m=3 / n=2$ island accelerates so that at the end of the run, the island width is approximately $0.3 \mathrm{a}$ and is comparable to that of the $m=2 / n=1$ island; although at the beginning of the run the $m=3 / n=2$ is a factor of three smaller than the $m=2 / n=1$ island. Recall that in the two-dimensional run the $m=3 / n=2$ saturates at about 0.1 a. Third, the growth rate of the $m=5 / n=3 i s l a n d$ also accelerates so that at the end of the run, its width is about $0.15 \mathrm{a}$. Fourth, although the width of both the $m=3 / n=2$ and $m=2 / n=1$ islands is about 0.3 a. at the end of the run, the radial extent to these islands when superimposed is about $0.5 \mathrm{a}$, which is about $25 \%$ larger than the width at the $\mathrm{m}=2 / \mathrm{n}=1$ island at the same time in the MASS code run.

The radial extent of the mode activity can also be ascertained from the velocity flow pattern in the $\zeta=0$ plane, as depicted in Fig. 14, where the velocity vectors are plotted at selected grid points for four different times. The length of the arrows is proportional to the magnitude of the velocity at a given time; the scale must be changed from frame to frame because the magnitude of the velocity changes by several orders of magnitude. Early' in time, when the $m=2 / n_{1}=1$ mode is present, one observes four vortices corresponding to an $m=2$ structure in the $\zeta=0$ plane. At the end of the run, however, when the $m=2 / n=1$ and $m=3 / n=2$ modes are comparable, the vortex structure is much more complicated because of the superposition of the two modes. 
As mentioned previously, the limitation on the length of time which the three-dimensional code can be run is due to the sharp deformations that develop in the toroidal current density due to the presence of several islands: In Fig. 15, the toroidal current density as a function of. $r$ in the $\zeta=0$ plane is plotted along the $\theta=-\pi$ and $\theta=0$ rays at the times indicated by the arrows in Figs. 5, 7, 8, and 12 . The $q=3 / 2$ and $q=2$ singular surfaces of the equilibrium are indicated by the vertical dotted lines; the $q=5 / 3$ singular surface is not indicated because it is very close to the $q=2$ surface $\left(r_{s 2}=0.7\right.$ a and $\left.r_{s} 53=0.62 \mathrm{a}\right)$. At $t=1.78 \times 10^{-2} \tau_{R}$, when the $m=2 / n=1$ is still the dominant mode, the deformation is similar to the current density deformation in the MASS code run (see Fig. 4). Later in time, however, additional spikes develop as the other islands become large; notice particularly the sharp deformation at the $x$-point of the $m=3 / n=2$ island.

For smaller values of $S$, the severe sharpness of the spike in the toroidal current density decreases because of the increased resistive diffusion. Consequently, in order to increase the effective length of time over which the deformation in the current density can be followed, we have also run the same $q$ profile at $S=3 \times 10^{3}$, i.e., at an effective $S$ value of $2 \times 10^{4}$ at $r=0$. In Fig. 16, the toroidal current density at $t=1.16 \times 10^{-2} \tau_{R}$ is presented; the deformation is quite severe. Near the end of this run, magnetic islands of many different helicities are present. In Fig. 17, the flux contours for twelve different helicities are presented at $t=1.08 \times 10^{-2} \vec{\tau}_{R}$. The importance of Fig. 17 is that it shows that if we include the $m=1 / n=1$ deformation in the flux contours, then the region of island or ergodic ${ }^{26}$ activity extends 
essentially from the plasma center to the limiter. Presumably this deformation of the field lines would lead to the rapid transport of heat from the plasma center to the limiter. ${ }^{26}$

We also ran the preceding case with different initial conditions and obtained similar results. In addition, our preliminary results for the flat profile with $q(0)=1.08$ also indicate that the odd m modes are strongly destabilized nonlinearly. 


\section{CONCLUSION}

The preceding results clearly indicate that in order to study the nonlinear growth of the tearing modes for flat $q$ profiles in cylindrical geometry, a full three-dimensional analysis must be employed. For a flat profile with $q(0)=1.37$, the $m=3 / n=2$ tearing mode, which is linearly unstable, is strongly destabilized nonlinearly by the $m=2 / n=1$ mode. Other modes are also nonlinearly destabilized, particularly the $m=1 / n=1$ mode. Due to the development of islands of many different helicities, the toroidal current density is severely deformed. The corresponding region of island activity or ergodicity can extend essentially from the plasma center to the limiter. Presumably, this deformation of the field lines can lead to rapid transport of heat and particles from the plasma center to the limiter.

The destabilization of the $m=3 / n=2$ mode is significant because it may correspond to the recent experimental observations from PLT. In many disruptive PLT discharges, the disruption appears to be centered about a surface that is inside the $q=2$ surface and perhaps coincides with the $q=3 / 2$ surface. In addition, the experimentally observed vertical asymmetry in the disruption could be explained by the presence of an $m=3 / n=2$ mode. If we assume that the modes are rotating toroidally so that the frequency of a mode is proportional to the toroidal mode number, then attributing the asymmetry to an $m=3 / n=2$ can explain the observation that the frequency associated with the asymmetry is twice that of the $m=2 / n=1$ precursor oscillation. 


\section{ACKNOWLEDGEMENT}

Two of the authors (H. R. H. and B. V. W.) thank Marshall N. Rosenbluth for his help in the formulation of this work. 


\section{REFERENCES}

1. E. P. Gorbunov and K. A. Razumova, Sov. Atomic Energy 15, 1105-1112 (1963): L. A. Artsimovich, S. V. Mirnov, and V. S. Strelkov, Sov. Atomic Energy 17, 886-893 (1964); V. S. Vlasenkov et al., Tokyo IAEA Conf., Nucl. Fusion Suppl., 1-5 (1974).

2. F. Karger et al., Plasma Physics and Controlled Nuclear Fusion Research 1976, Vol. 1, p. 267 (1977).

3. Equipe TFR, Plasma Physics and Controlled Nuclear Fusion Research 1976, Vol. 1, p. 279 (1977).

4. N. R. Sauthoff, S. von Goeler, and W. Stodiek, "X-Ray Fluctuation Measurements on the PLT Tokamak," Section 5.1, Status of Ohmic Heating in PLT, Princeton Plasma Physics Laboratory, Princeton University, Princeton, New Jersey (September 1977).

5. B. V. Waddell, G. Laval, and.M. N. Rosenbluth, Reduction of the Growth Rate of the $m=1$ Resistive Magneto-Hydrodynamic Mode by Finite Gyroradius Effects, ORNL/TM-5968, Oak Ridge National Laboratory, Oak Ridge, Tennessee (July 1,977); G. Ara, B. Basu, B. Coppi, G. Laval, M. N. Rosenbluth, and B. V. Wadde11, "Magnetic Reconnection and $m=1$ Oscillations in Current Carrýing Plasmas," submitted to Annals of Physics.

6. M. N. Bussac, D. Edery, R. Pellat, and J. L. Soule, Plasma Physics and Controlled Nuclear Fusion Research 1976, Vo1. 1, p. 607 (1977).

7. H. P. Furth, J. Killen, and M. N. Rosenbluth, Phys. Fluids $\underline{6}, 459$ (1963).

8. H. P. Furth, P. H. Rutherford, and H. Selberg, Phys. Fluids 16,1054 (1973). 
9. M. N. Rosenbluth, D. A. Monticello, H. Strauss, and R. B. White, Phys. Fluids 19, 1987 (1976).

10. B. Coppi, R. Galvao, R. Pellat, M. N. Rosenbluth, and P. H. Rutherford, Fizika Plazmy $\underline{6}, 961$ (1976).

11. M. N. Rosenbluth, R. Y. Dagazian, and P. H. Rutherford, Phys. Fluids 16, 1894 (1973).

12. A. F. Danilov, Yu. N. Dnestrovski, D. P. Kostomarov, and A. M. Popov, Fizika Plazmy 2, 167 (1976).

13. A. Sykes and J. A. Weśšon, Phys. Rev. Lett. 37, 140 (1976).

14. B. V. Waddell, M. N. Rosenbluth, D. A. Monticello, and R. B. White, Nucl. Fusion $1 \underline{6}, 528$ (1976).

15. Masafumi Azumi, Japan Atomic Energy Research Institute, Tokai, Research Establishment, Tokai-Mura, Ibaraki-Ken, Japan, private communication (1976).

16. B. B. Kadomtsev, Fizika Plazmy 1, 710 (1975).

17. B. V. Wadde11, G. L. Jahns, J. D. Callen, and H. R. Hicks, Internal Disruptions in Tokamaks, ORNL/TM-5840, Oak Ridge National Laboratory, Oak Ridge, Tennessee (May 1977).

18. P. H. Rutherford, Phys. Fluids 16, 1903 (1973).

19. R. B. White, D. A. Monticello, M. N. Rosenbluth, and B. V. Waddell, Plasma Physics and Controlled Nuclear Fusion Research 1976, Vol. 1, p. 569 (1977).

20. D. Biskamp and H. Welter, Plasma Physics and Controlled Nuclear Fusion Research 1976, Vol. 1, p. 579 (1977). 
21. R. B. White, D. A. Monticello, and M. N. Rosenbluth, "Simulation of a Major Tokamak Disruption," PPPL-1364, Princeton P. lasma Physics Laboratory, Princeton University, Princeton, N. J. (July, 1977).

22. 'S. von Goeler, Proc. 7th European Conference on Controlled Fusion and plasma Physics, Vol. II, p. 71 (1975).

23. H. R. Strauss, Phys. Fluids 19, 134 (1976).

24. B. V. Wadde11, M. N. Rosenbluth, D. A. Monticello, R. B. White, and B. Carreras, "Nonlinear Numerical Algorithms for Studying Tearing Modes," Lecture presented at the Trieste College in Theoretical and Computational Plasma Physics, Trieste, Italy (March 22-April 9, 1977), to be published by the IAEA.

25. H. P. Furth, Propagation and Instabilities in Plasmas, ed. by W. I. Futterman, Stanford University Press, Stanford, California (1973), pp. 87-102.

26. T. Stix, Phys. Rev. Lett. $\underline{36}$, (1976) 521. 
32

ORNL/OWG/FED - 77548

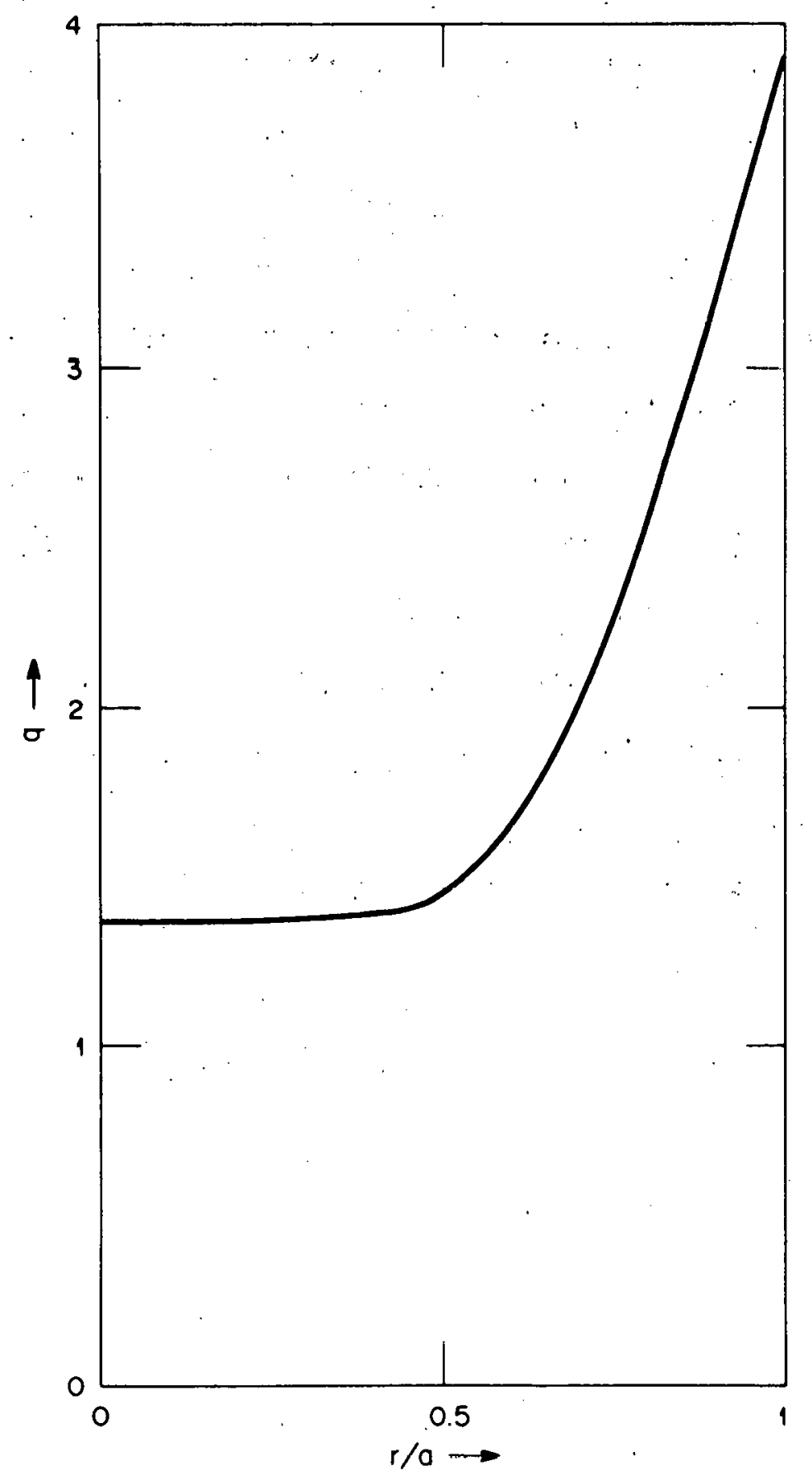

Fig. 1. Safety factor $q$ as a function of $r / a$ (flat current model). 


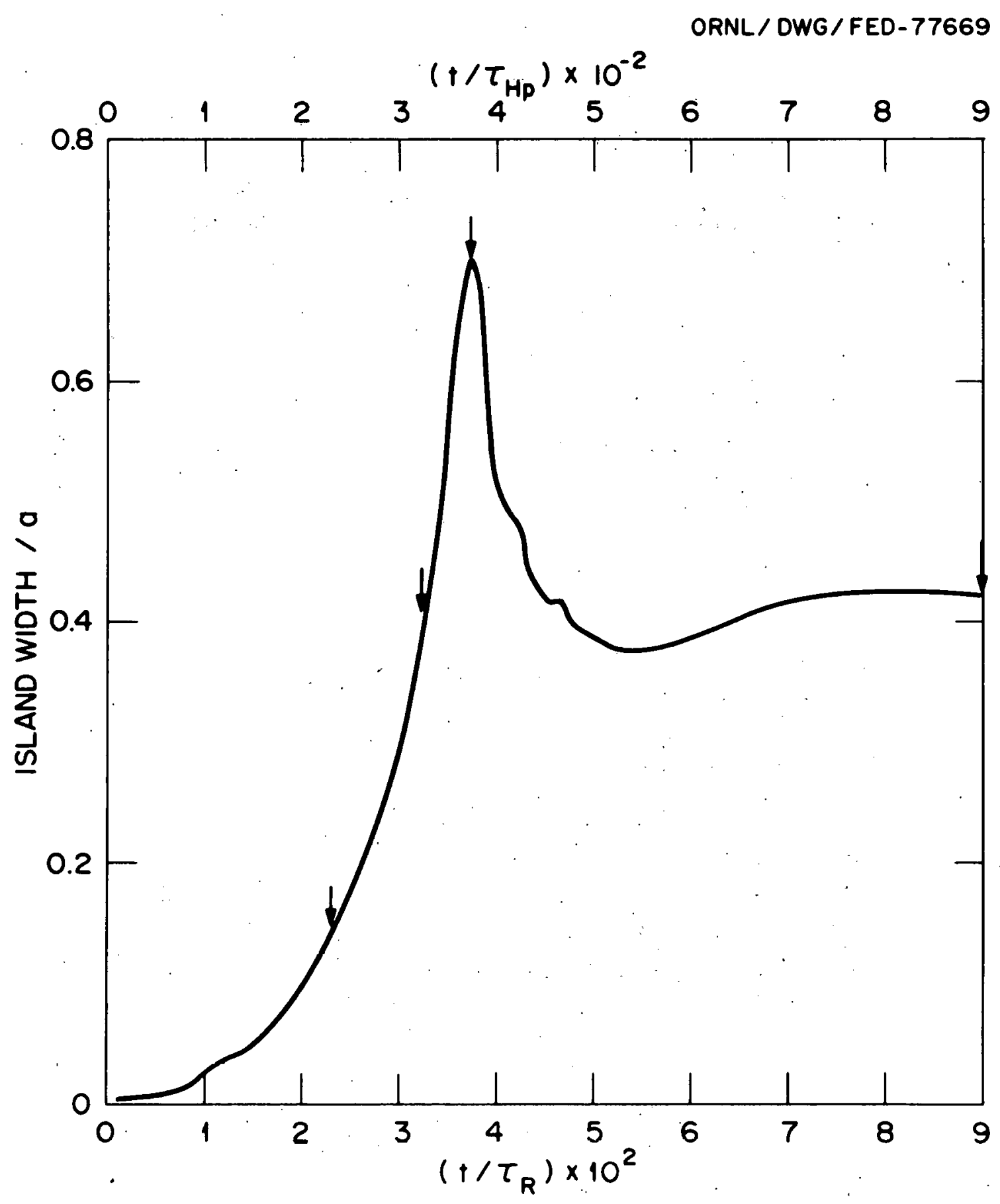

Fig. 2. Width of the $m=2 / n=1$ magnetic island as a function of time (from the two dimensional code). 

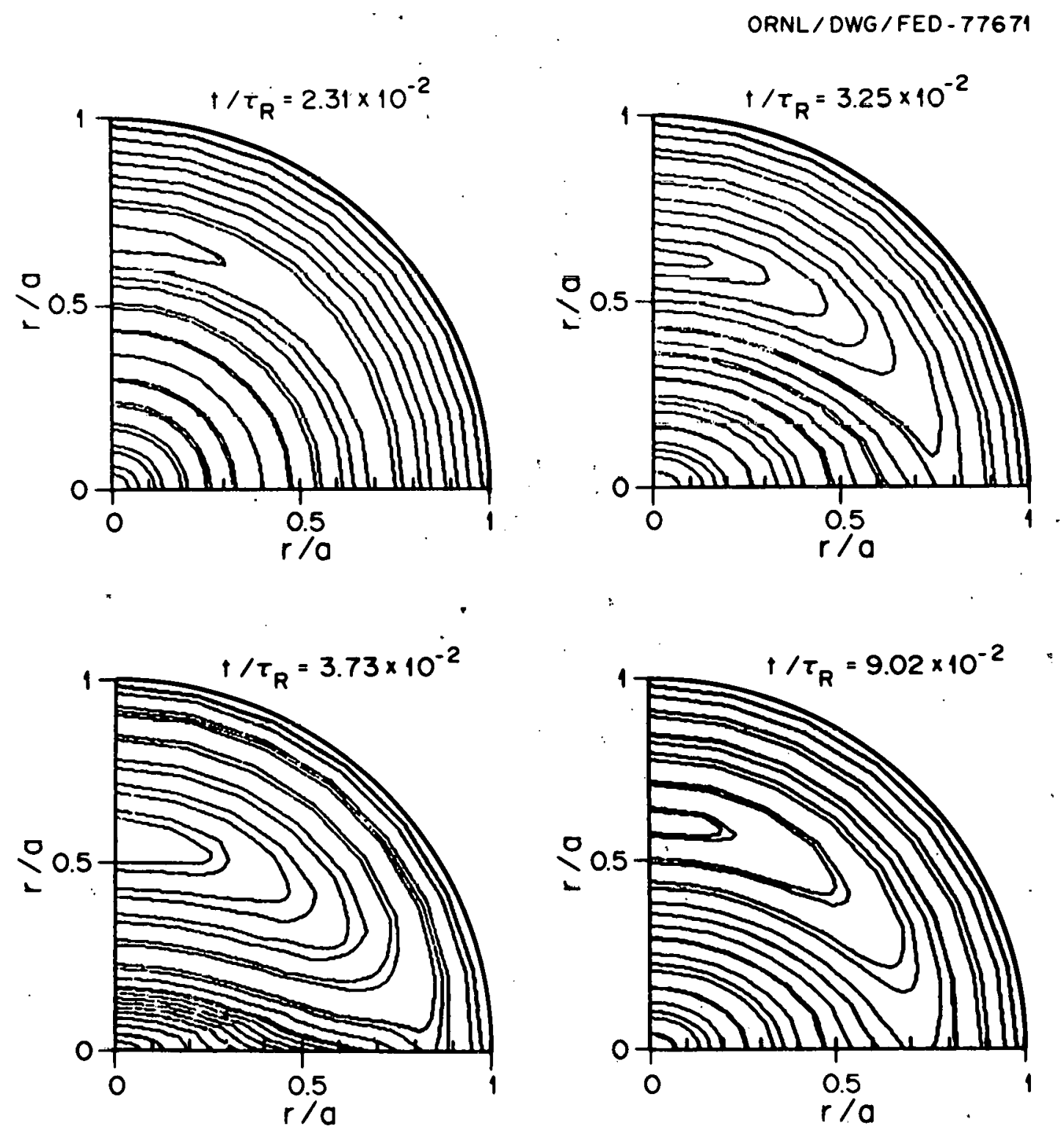

Fig. 3. Helical flux contours for the $m=2 / n=1$ mode at the time indicated by the arrows in Fig. 2 (from the two-dimensional code). 

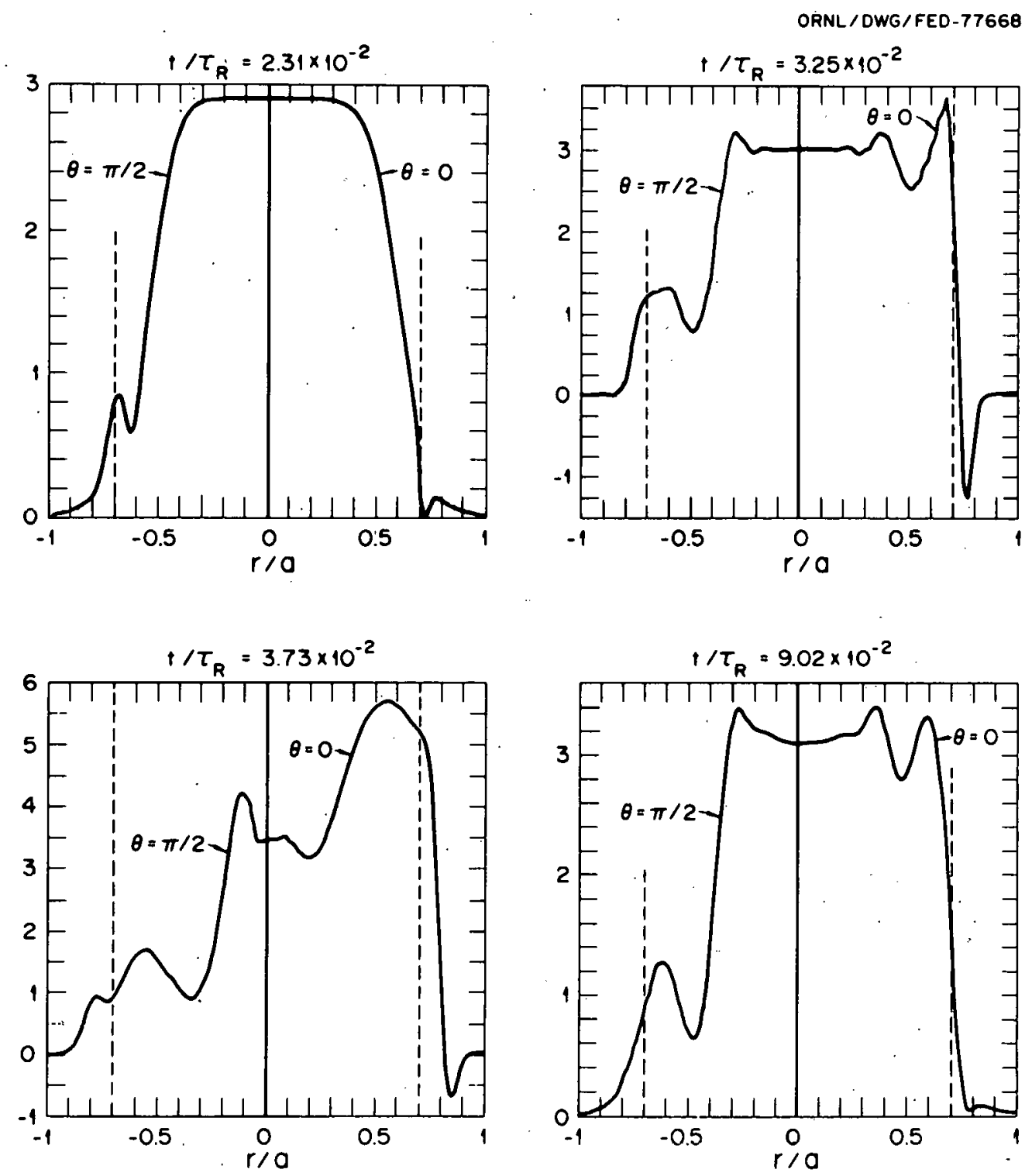

Fig. 4. Toroidal current density as function of $r / a$ at the times indicated by the arrows in Fig. 2 (from the two-dimensional code). 


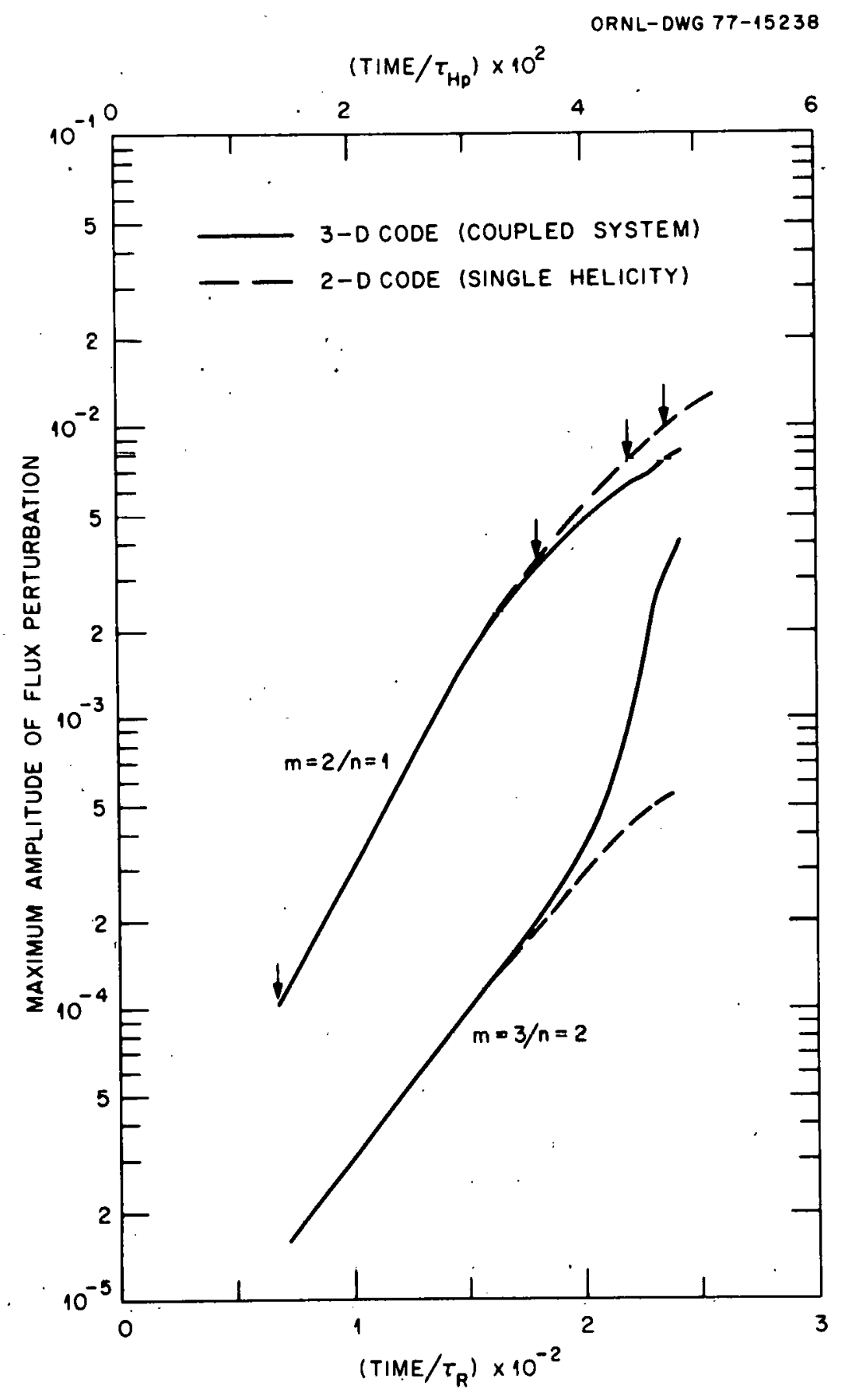

Fig. 5. Time evolution of the maximum ampilitudes of the flux perturbations of various modes. 


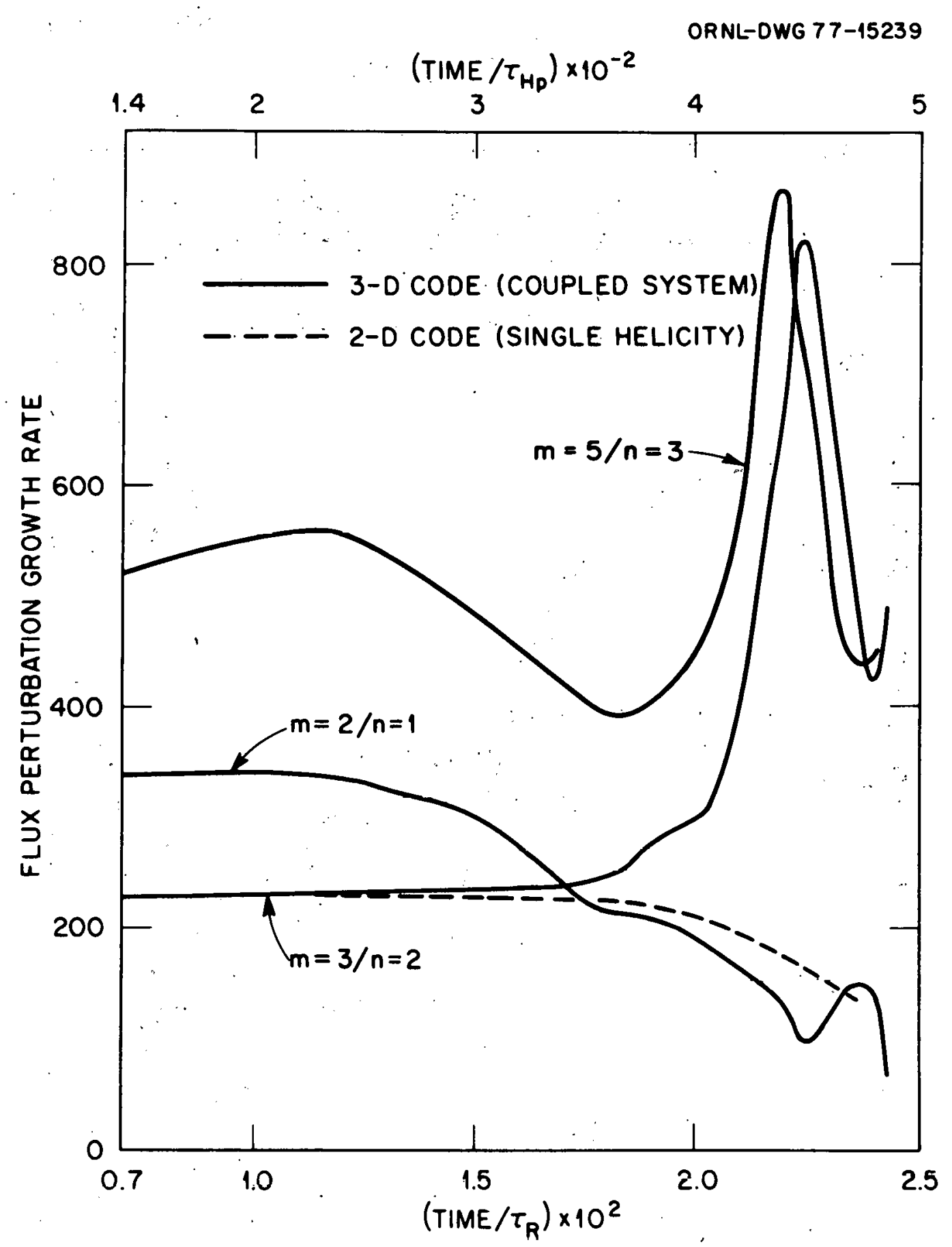

Fig. 6. Time evolution of the growth rates of the flux perturbations of various modes. 


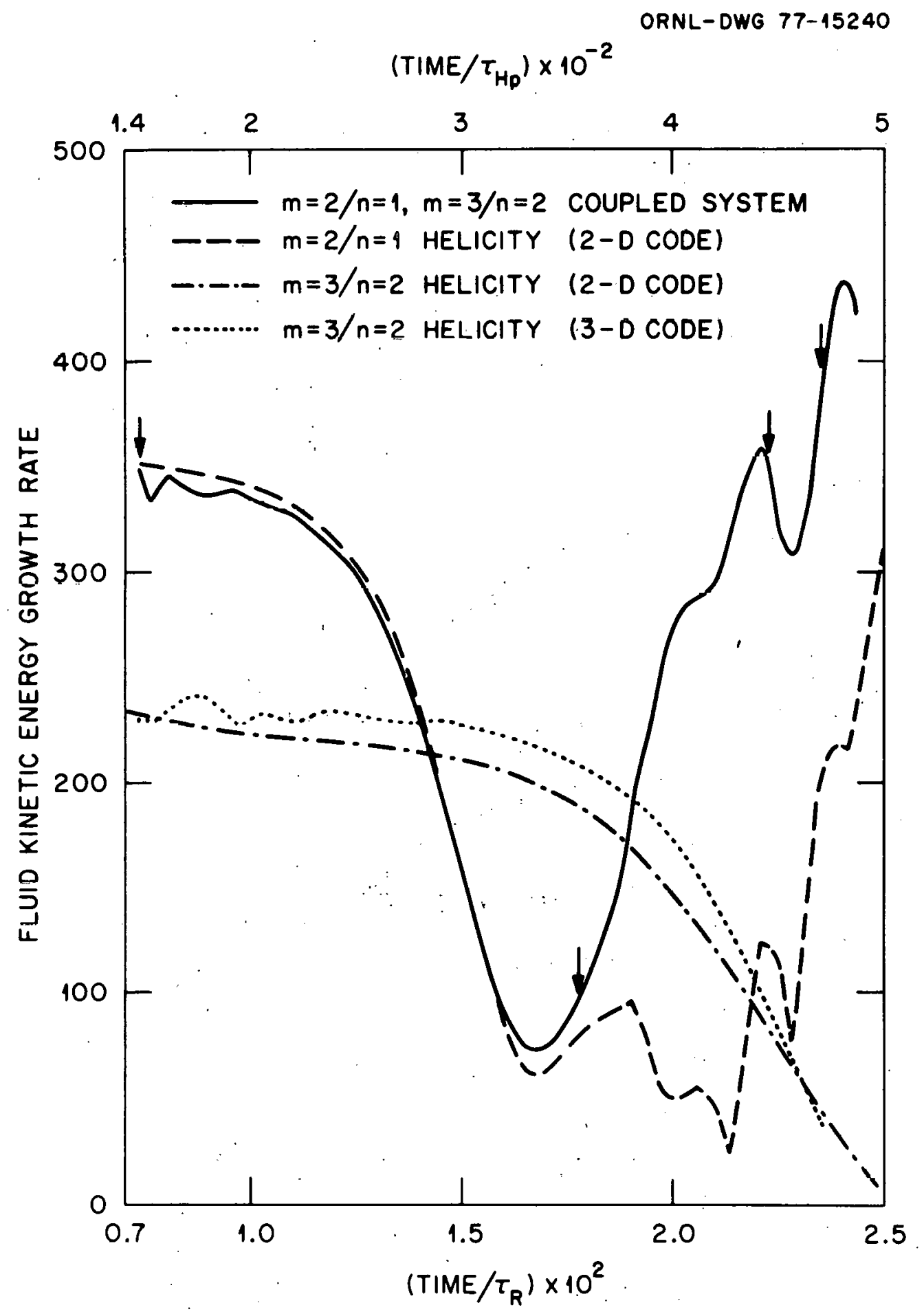

Fig. 7. Time evolution of the total fluid kinetic energy growth rate for various runs. 


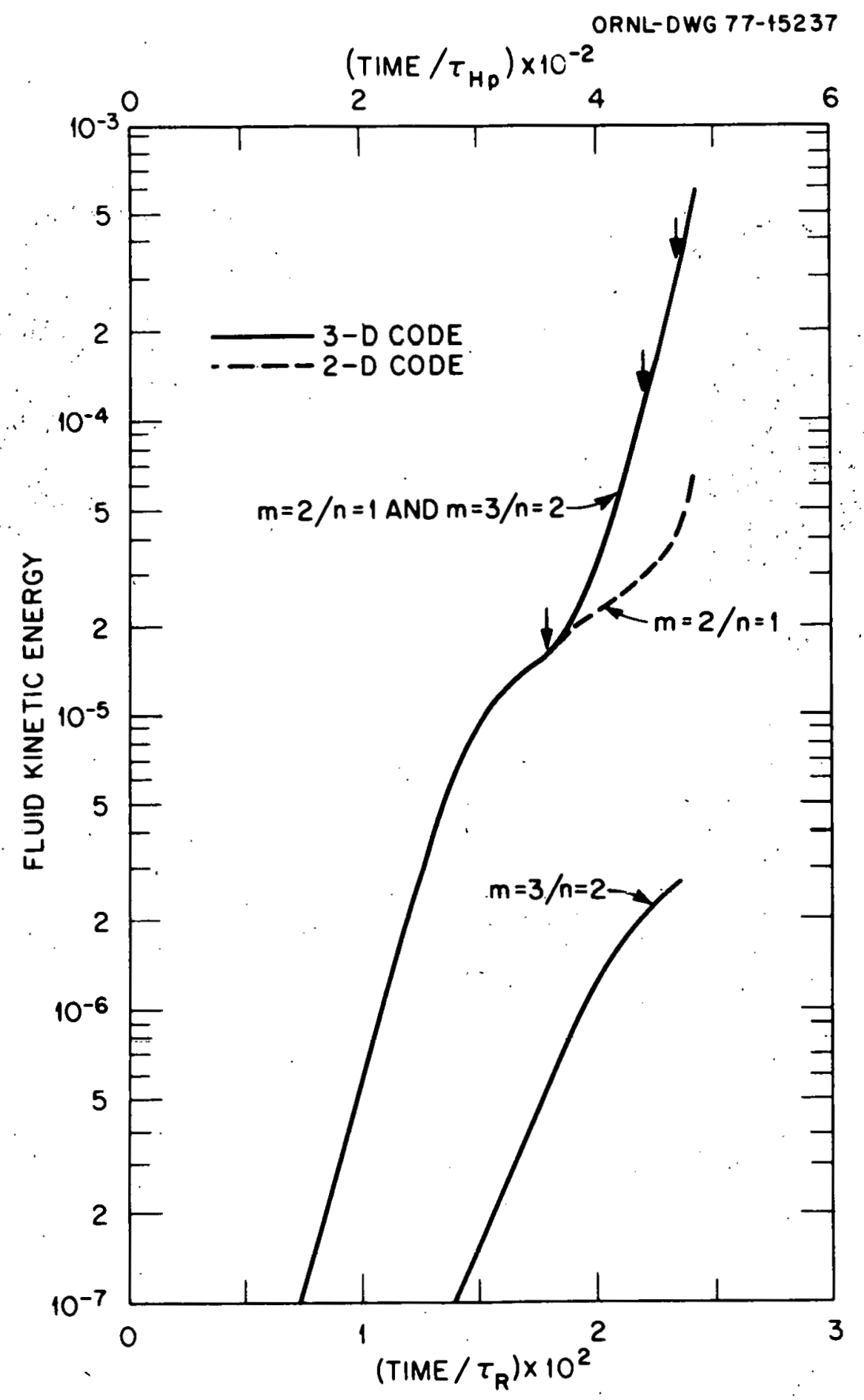

Fig. 8. Time evolution of the total fluid kinetic energy for various runs. 
ORNL/OWG/FED-77467
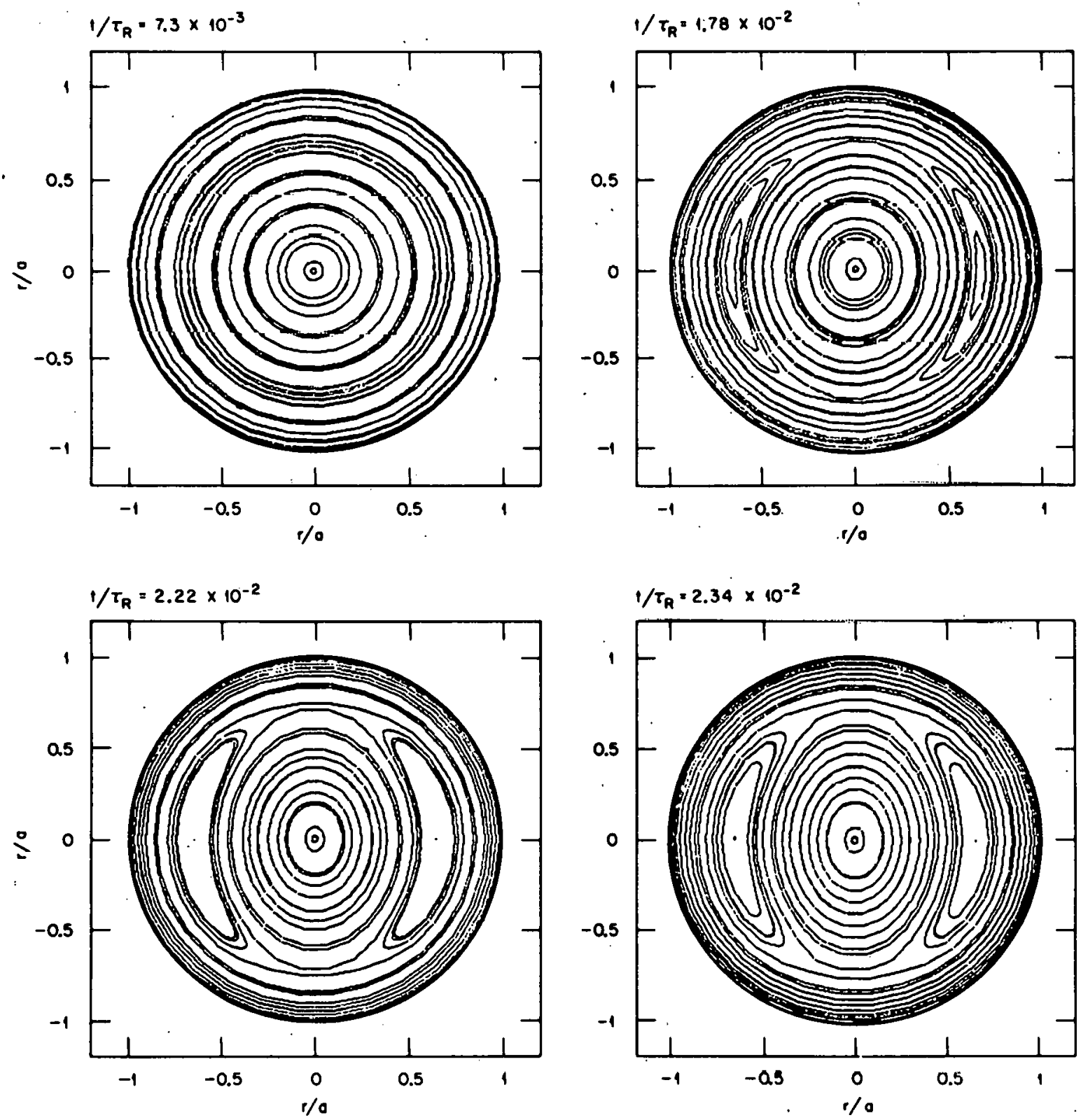

Fig. 9. The $m=2 / n=1$ helical flux contours at the times indicated by the arrows in Figs. 5,7 ; and 8 . 

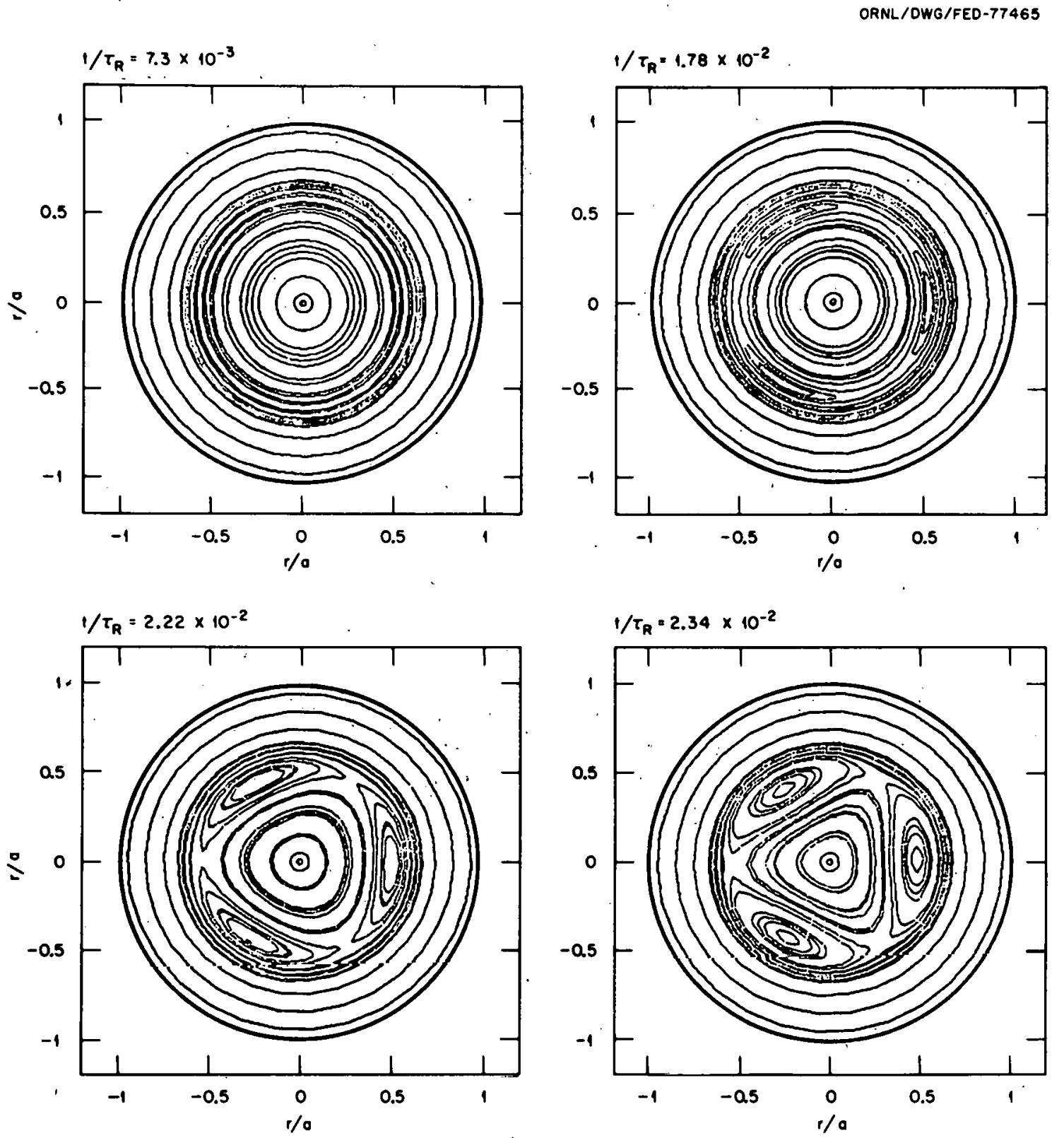

Fig. 10. The $m=3 / n=2$ helical flux contours at the times indicated by the arrows in Figs. 5, 7, and 8 . 

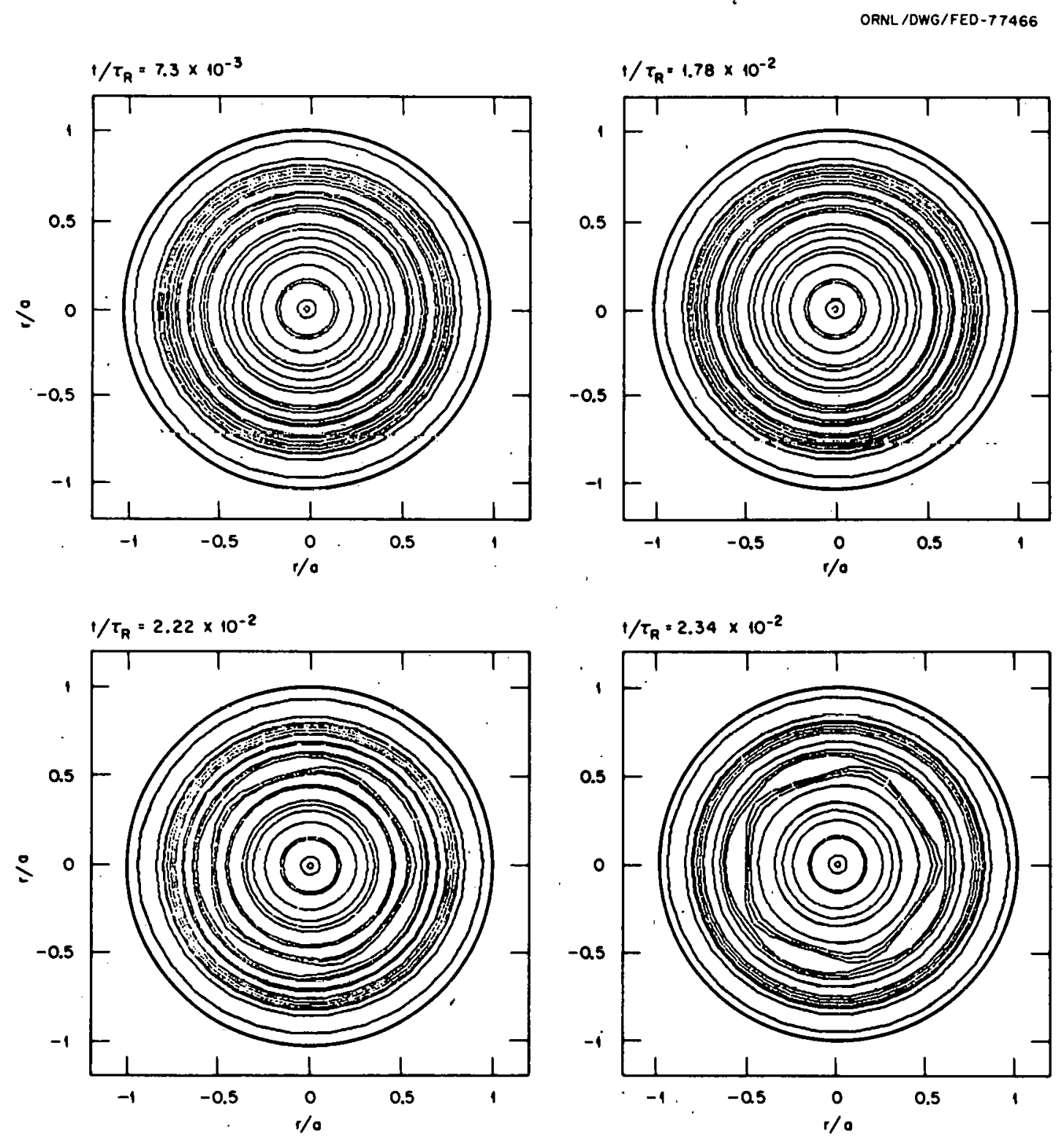

Fig. 11. The $m=5 / n=3$ helical flux contours at the times indicated by the arrows in Figs. 5,7 , and 8. 

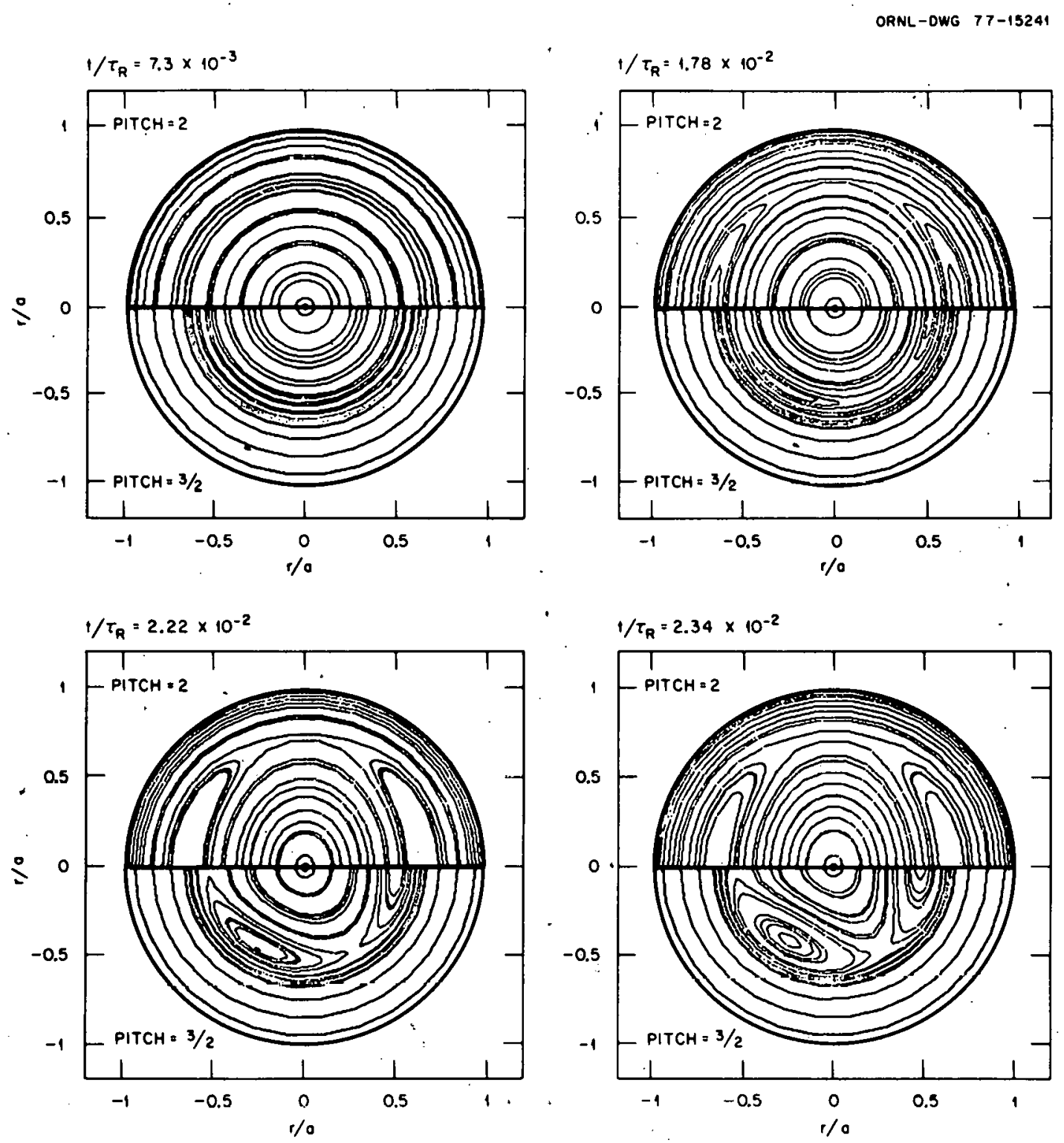

Fig. 12. The $m=2 / n=1$ (upper half plane) and the $m=3 / n=2$ (lower half plane) helical flux contours at the times indicated by the arrows in Figs. 5,7 , and 8 . 


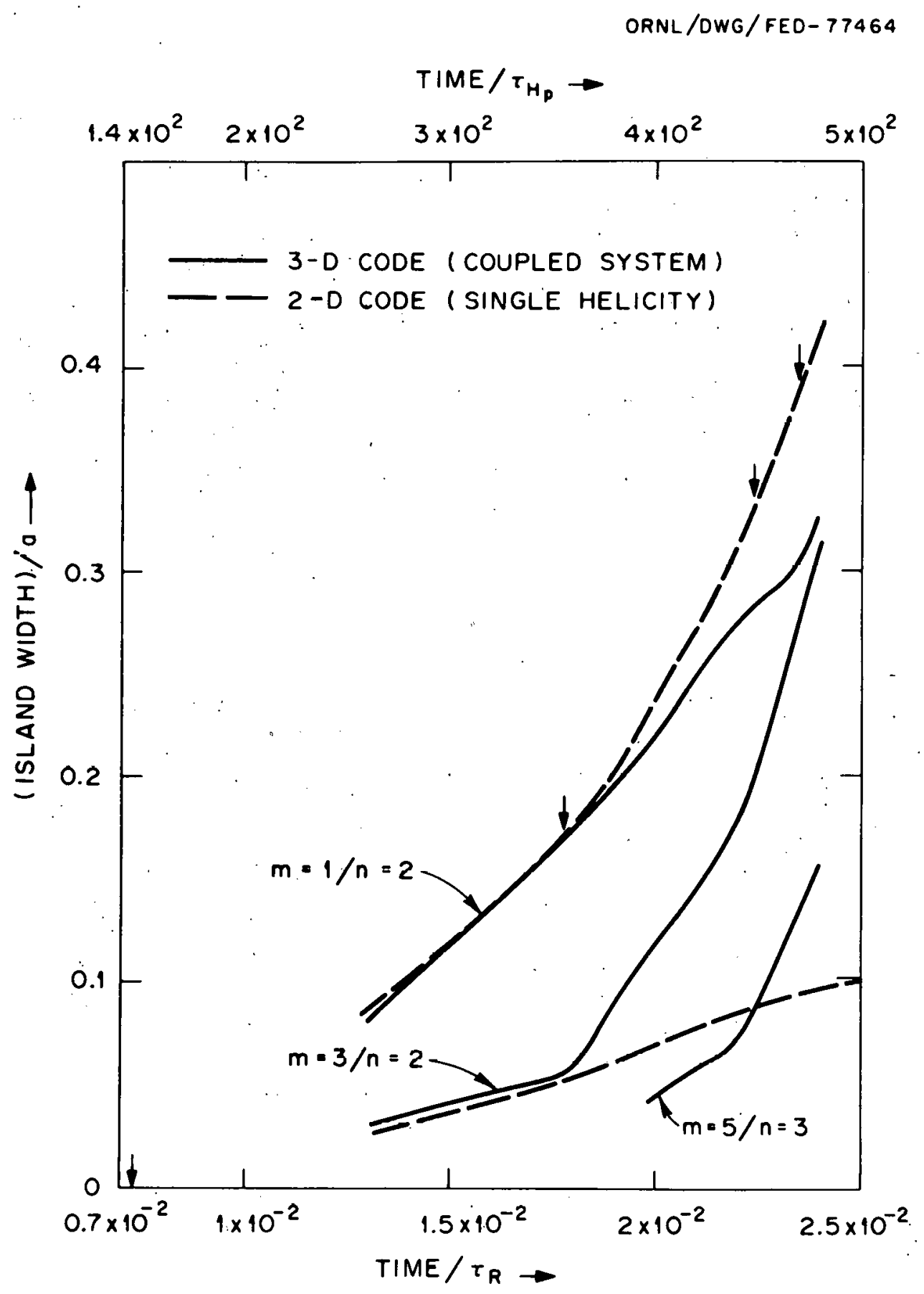

Fig. 13. Selected magnetic island widths as a function of time. 

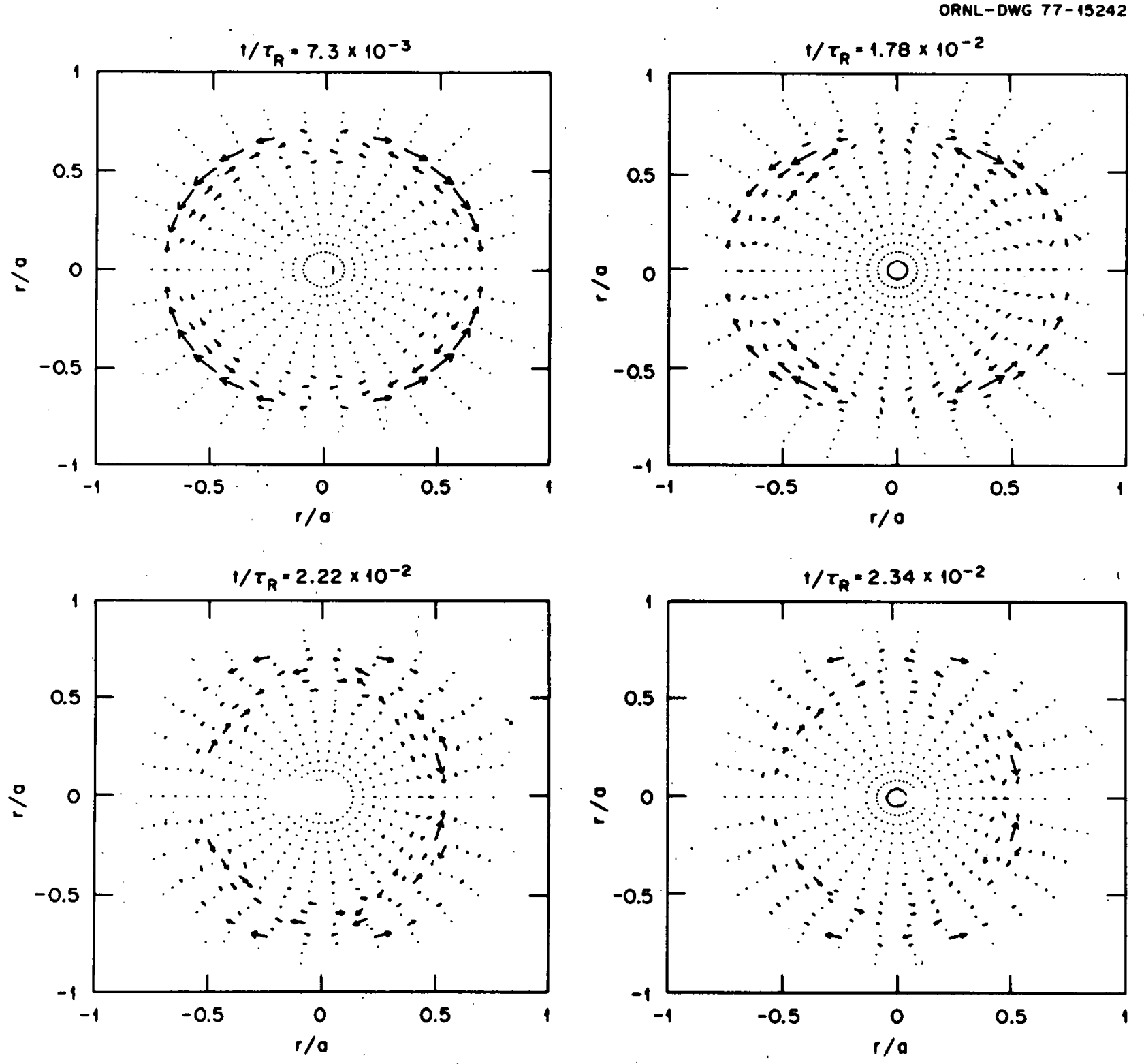

Fig. 14. The velocity flow at the times indicated by the arrows in Figs. 5,7 , and 8 . 
ORNL-DWG 77-15236
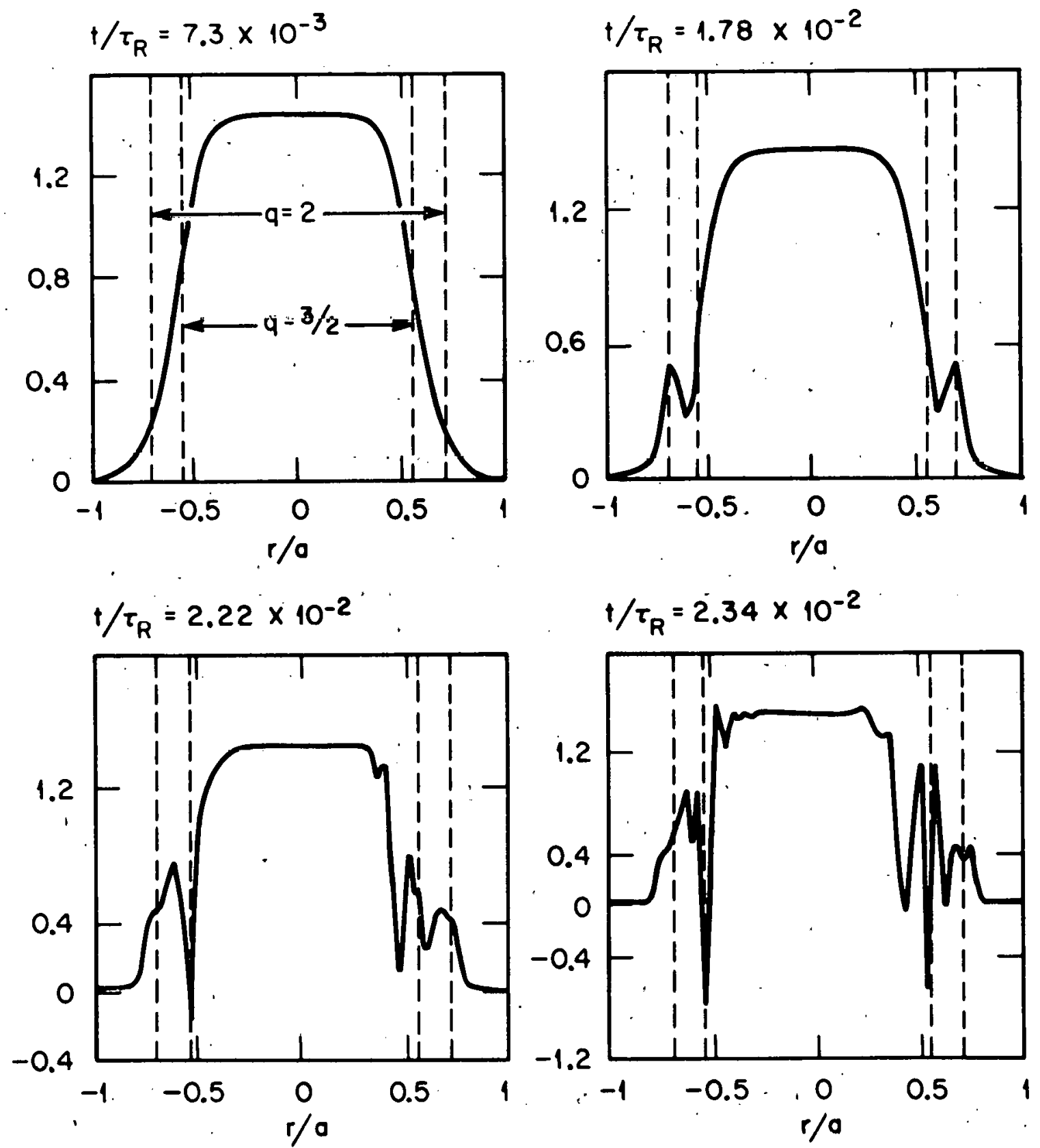

Fig. 15. The toroidal current density (with a minus sign) as a function of $\mathrm{r} / \mathrm{a}$ at the times indicated by the arrows in Figs. 5, 7, and 8 . 


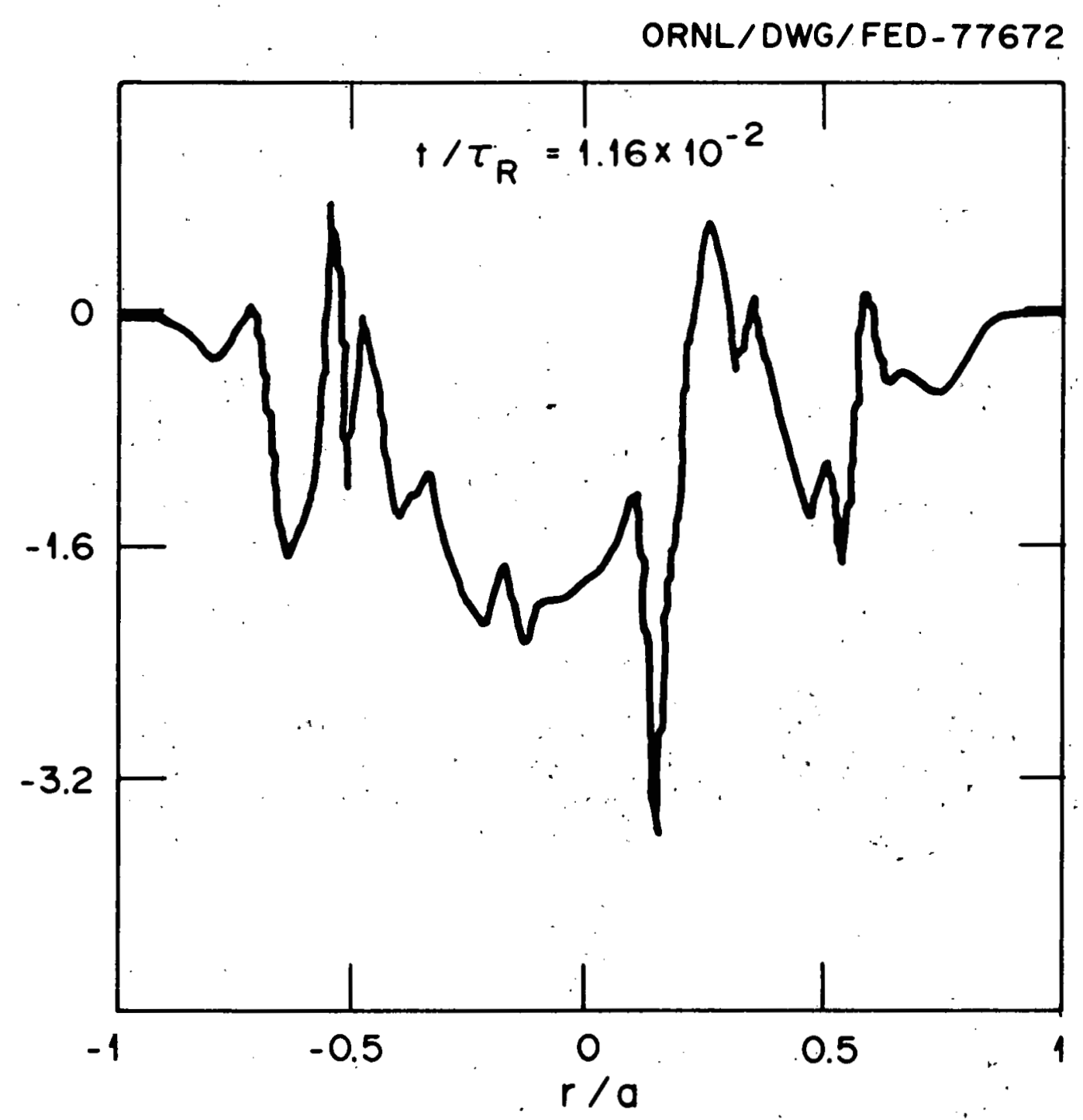

Fig. 16. The toroidal current density for $S=3 \times 10^{3}$ at $t=$ $1.16 \times 10^{-2} \tau_{R}$. 

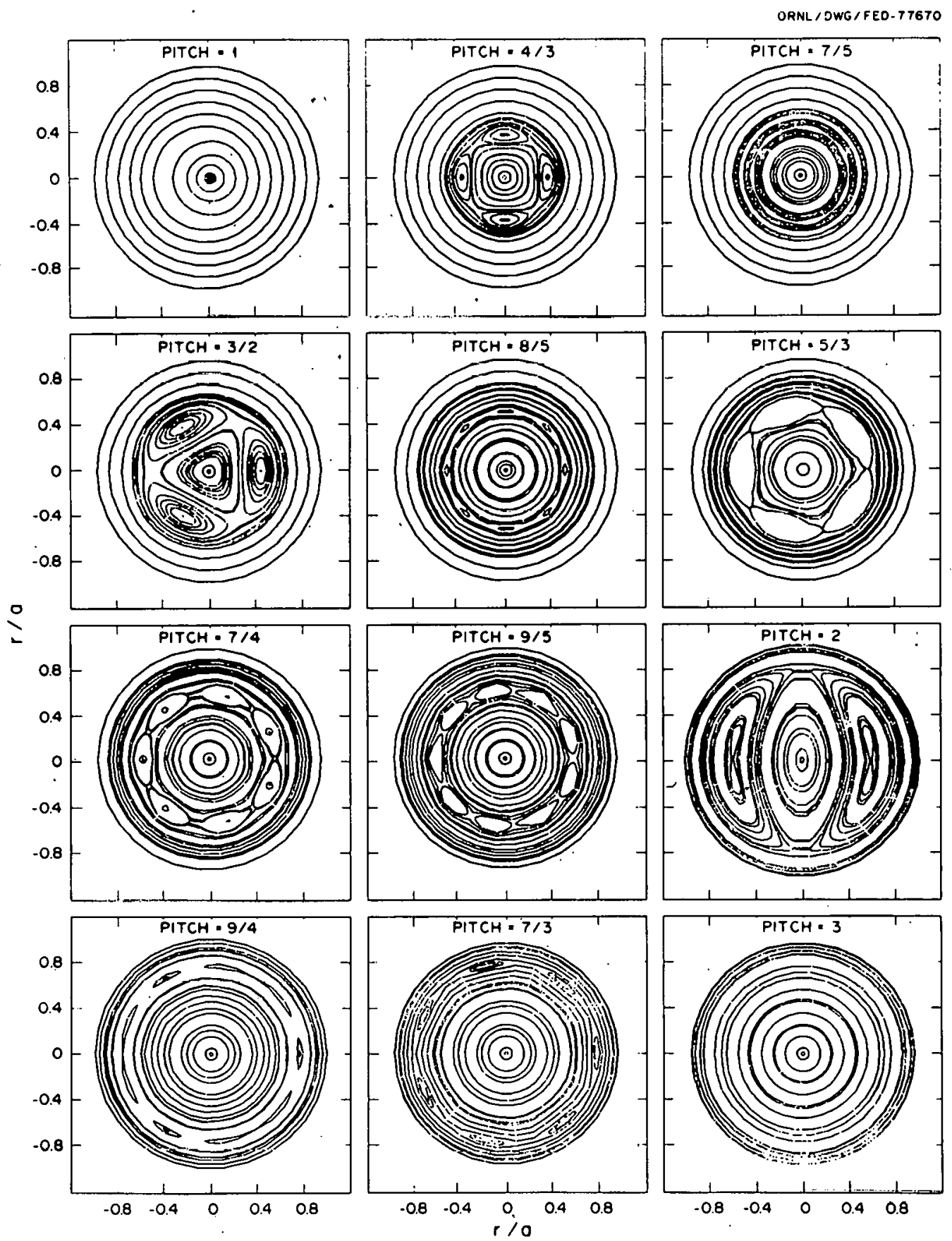

Fig. 17. Helical flux contours of different pitch for $S=3 \times 10^{3}$. at $t=1.08 \times 10^{-2} \tau_{R}$. 
INTERNAL DISTRIBUTION

ORNL/TM-6096

1. J. D. Caḷlen

2. J. F. Clarke

3. R. A. Dory

4. G. G. Kelley

5. 0. B. Morgan

6. M. W. Rosenthal

7-31. B. V. Waddell

32-34. Laboratory Records Department

35. Laboratory Records, ORNL - RC

36. Y-12 Document Reference Section

37-38. Central Research Library

39. Fusion Energy Division Library

40. Fusion Energy Division Reports Office

41. ORNL Patent Office

\section{EXTERNAL DISTRIBUTION}

42. Plasma Physics Library, Plasma Physics Laboratory, Princeton Univ., Forrestal Campus, P.0. Box 451, Princeton, NJ 08540

43. Controlled Thermonuclear Research Library, Lawrence Livermore Laboratory, P.0. Box 808, Livermore, CA 94550

44. Q Division Library, Los Alamos Scientific Laboratory, P.0. Box 1663, Los Alamos, NM 87544

45. Controlled Thermonuclear Research Library, c/o Weston M. Stacey, Jr., Argonne National Laboratory, 9700 S. Cass Ave., Argonne, IL 60439

46. CTR Computer Center, c/o Dr. John Killeen, Lawrence Livermore Laboratory, P.0. Box 808, Livermore, CA 94550

47. Librarian, Culham Laboratory, U.K. Atomic Energy Authority, Abingdon, Oxon, 0x14 3DB, United Kingdom

48. Ruth Lengye, Bibliothek, Max-Planck Institut für Plasmaphysik, 8046 Garching bei München, Federal Republic of Germany

49. Library, Centre de Recherches en Physique des Plasmas, 21 Avenue des Bains, 1007, Lausanne, Switzerland

50. A. M. Dupas, Documentation S.I.G.N., Départment de la Physique du Plasma et de la Fusion Controlée, Association EURATOM-CEA sur la Fusion, Centre d'Etudes Nucléaires, BP 85 Centre Du TRI 38041 Grenoble Cedex (France)

51. Bibliotheque, Service du Confinement des Plasmas, C.E.A., B.P. No. 6, 92, Fontenay-aux-Roses (Seine) France

52. Library, International Centre for Theoretical Physics, Trieste, Italy

53. Library, Laboratorio Gas Ionizzati, Frascati, Italy

54. V. E. Ivanov, Physical-Technical Institute of the Ukranian Academy of Sciences, Sukhumi, U.S.S.R.

55. L. M. Kovrizhnikh, Lebedev Institute of Physics, Academy of Sciences of the U.S.S.R., Leninsky Prospect 53, Moscow, U.S.S.R.

56. Prof. Dshumber G. Lominadze, Academy of Sciences of the Georgian SSR, 8 Dzerzhinski St., 38004 , Tbilisi, U.S.S.R.

57. Library, Inst. for Plasma Physics, Nagoya Univ., Nagoya, Japan 464 
58. Library, FOM-Institut voor Plasma-Fysica, Rijnhuizen, Jutphaas, Netherlands

59. Plasma Physics Group, Department of Engineering Physics, Australian National University, P.0. Box 4, Canberra A.C.T. 2600, Australia

60. Thermonuclear Library, Japan Atomic Energy Research Institute, Tokai, Naka, Ibaraki, Japan

61. Dr. D. G. McAlees, Exxon Nuclear Co., Inc., Research \& Technology Laser Enrichment Department, 2955 George Washington Way, Richland, WA 99352

62. CTR Reading Room, c/o Prof. D. W. Kerst, Dept. of Physics, Sterling Hall, Univ. of Wisconsin, Madison, WI 53706

63. CTR Reading Room, c/o Prof. I. B. Bernstein, Yale Univ., 200 Mason Laboratory, Dept. of Engineering \& Applied Science, New Haven, CT 06510

64. Center for Plasma Physics and Thermonuclear Research, c/o D. W. Russ, Physics Dept., Univ. of Texas, Austin, TX 78712

65. CTR Reading Room, c/o Prof. B. D. Fried, Physics Dept., Univ. of Lalitornía, Los Âryeles, CA 90024

66. CTR Reading Room, C/o Prof. David C. Montgomery, Physics \& Astronomy Dept., Univ. of Iowa, Iowa City, IA 52240

67. Magneto-Fluid-Dynamics Library, c/o Dr. Harold Grad, Courant Inst. of Math. Sci., New York Univ., 251 Mercer St., New York, NY 10012

68. CTR Reading Room, C/o Prof. Allan N. Kaulman, Physics Dcpt., Univ. of California, Berkeley, CA 94720

69. Dr. David A. Dingee, Fusion Programs, Battelle-Northwest, Battelle Boulevard, Richland, WA 99352

70. CTR Reading Room, c/o Prof. C. S. Liu, Dept. of Physics and Astronomy, Univ. of Maryland, College Park, MD 20742

71. CTR Reading Room, c/o Prof. T: Kammash, 103 Research Admin. Bldg., N. Campus, Univ. of Michigan, Ann Arbor, MI 48105

72. CTR Reading Room, c/o Dr. Ravi N. Sudan, Phillips Hall, Cornell Univ., Ithaca, NY 14850

73. Prof. Marshall N. Rosenbluth, Institute for Advanced Study, Princeton, NJ 08540

74. CTR Reading Room, c/o Prof. R. Gross, Plasma Research Lab., Columbia Univ., New York, NY 10027

75. CTR Reading Room, c/o Prof. Roy Gould, California Inst. of Tech., M.S. 116-81, Pasadena, CA 91125

76. Dr. Nicholas A. Krall, Science Applications, Inc., P.0. Box 2354, 1200 Prospect St., La Jolla, CA 92037

77. CTR Reading Room, c/0 Dr. Jay P. Boris, Plasma Physics, Naval Research Laboratory, Washington, D.C. 20390

78. Professor A. Simon, Dept. of Mechanical \& Aerospace Sciences, University of Rochester, Rochester, NY 14627

79. CTR Library, c/o Dr. Alan F. Haught, United Technologies Research Labs, East Hartford, CT 06108

80. Dr. H. K. Forsen, Exxon Nuclear Co., Inc., 777-106th Avenue, NE, C-000777, Bellevue, WA 98009

81. Dr. George Vahala, Physics Dept., College of William \& Mary, Williamsburg, VA 23185

82. Dr. Robert E. Price, Division of Magnetic Fusion Energy, G-234, Department of Energy, Washington, D.C. 20545 
83. Dr. R. C. Davidson, Division of Magnetic Fusion Energy, G-234 Department of Energy, Washington, D.C. 20545

84. Dr. Oscar P. Manley, Division of Magnetic Fusion Energy, G-234, Department of Energy, Washington, D.C. 20545

85. Mr. E. E. Kintner, Division of Magnetic Fusion Energy, G-234, Department of Energy, Washington, D.C. 20545

86. Dr. L. D. Pearlstein, L-388, Lawrence Livermore Laboratory, P.0. Box 808, Livermore, CA 94550

87. Dr. J. P. Friedberg, Los Alamos Scientific Laboratory, Los Alamos, NM 87544

88. Dr. David J. Rose, Dept. of Nuclear Engineering, MIT, Cambridge, MA 02139

89. Dr. Gareth E. Guest, General Atomic Co., P.0. Box 81608, San Diego, CA 92138

90. Dr. Claude Mercier, Service du Theorie des Plasmas, Centre d'Etudes Nucléaires, Fontenay-aux-Roses (Seine) France

91. Dr. J. B. Taylor, Culham Laboratory, UKAEA, Abingdon, Oxon, 0X14 3DB, United Kingdom

92. Dr. D. Pfirsch, Institute for Plasma Physics, 8046 Garching bei München, Federal Republic of Germany

93. Dr. V. D. Shafranov, I. V. Kurchatov Inst. of Atomic Energy, 46 Ulitsa Kurchatova, P.0. Box 3402, Moscow, U.S.S.R.

94. Dr. A. Rogister, Institute for Plasma Physics, KFA, Postfach 1913, D-5170, Jülich 1, Federal Republ ic of Germany

95. Dr. J. G. Cordey, Culham Laboratory, UKAEA, Abingdon, Oxon, 0X14 3DB, United Kingdom

96. Dr. David Baldwin, L-388, Lawrence Livermore Lab., P.0. Box 808 , Livermore, CA 94550

97. CTR Reading Room, c/o Prof. Bruno Coppi, Dept. of Physics, MIT, Cambridge, MA 02138

98. Dr. Harold P. Furth, Princeton Plasma Physics Lab., Princeton Univ., P.0. Box 451, Princeton, NJ 08540

99. Dr. Paul H. Rutherford, Princeton Plasma Physics Lab., Princeton Univ., P.0. Box 451, Princeton, NJ 08540

100. Research \& Technical Support Div., Oak Ridge Operations, Department of Energy, P. O. Box E, Oak Ridge, TN 37830

101-127. Tech. Information Center, P. O. Box 62, Oak Ridge, TN 37830 